\title{
Vascular Inflammation and Oxidative Stress: Major Triggers for Cardiovascular Disease
}

\author{
Sebastian Steven, ${ }^{1,2}$ Katie Frenis, ${ }^{1}$ Matthias Oelze, ${ }^{1}$ Sanela Kalinovic, ${ }^{1}$ Marin Kuntic, \\ Maria Teresa Bayo Jimenez, ${ }^{1}$ Ksenija Vujacic-Mirski, ${ }^{1}$ Johanna Helmstädter, ${ }^{1}$ \\ Swenja Kröller-Schön, ${ }^{1}$ Thomas Münzel, ${ }^{1,3}$ and Andreas Daiber $\mathbb{D}^{1,3}$ \\ ${ }^{1}$ Center for Cardiology, Cardiology I-Laboratory of Molecular Cardiology, University Medical Center of the Johannes \\ Gutenberg-University, Mainz, Germany \\ ${ }^{2}$ Center for Thrombosis and Hemostasis (CTH), University Medical Center of the Johannes Gutenberg-University, \\ Mainz, Germany \\ ${ }^{3}$ German Center for Cardiovascular Research (DZHK), Partner Site Rhine-Main, Mainz, Germany
}

Correspondence should be addressed to Andreas Daiber; daiber@uni-mainz.de

Received 31 January 2019; Accepted 20 May 2019; Published 23 June 2019

Academic Editor: Luciano Saso

Copyright (c) 2019 Sebastian Steven et al. This is an open access article distributed under the Creative Commons Attribution License, which permits unrestricted use, distribution, and reproduction in any medium, provided the original work is properly cited.

\begin{abstract}
Cardiovascular disease is a leading cause of death and reduced quality of life, proven by the latest data of the Global Burden of Disease Study, and is only gaining in prevalence worldwide. Clinical trials have identified chronic inflammatory disorders as cardiovascular risks, and recent research has revealed a contribution by various inflammatory cells to vascular oxidative stress. Atherosclerosis and cardiovascular disease are closely associated with inflammation, probably due to the close interaction of inflammation with oxidative stress. Classical therapies for inflammatory disorders have demonstrated protective effects in various models of cardiovascular disease; especially established drugs with pleiotropic immunomodulatory properties have proven beneficial cardiovascular effects; normalization of oxidative stress seems to be a common feature of these therapies. The close link between inflammation and redox balance was also supported by reports on aggravated inflammatory phenotype in the absence of antioxidant defense proteins (e.g., superoxide dismutases, heme oxygenase-1, and glutathione peroxidases) or overexpression of reactive oxygen species producing enzymes (e.g., NADPH oxidases). The value of immunomodulation for the treatment of cardiovascular disease was recently supported by large-scale clinical trials demonstrating reduced cardiovascular mortality in patients with established atherosclerotic disease when treated by highly specific anti-inflammatory therapies (e.g., using monoclonal antibodies against cytokines). Modern antidiabetic cardiovascular drugs (e.g., SGLT2 inhibitors, DPP-4 inhibitors, and GLP-1 analogs) seem to share these immunomodulatory properties and display potent antioxidant effects, all of which may explain their successful lowering of cardiovascular risk.
\end{abstract}

\section{Inflammation and the Global Burden of Disease and Age-Related Cardiometabolic Complications}

1.1. Cardiovascular Risk Factors and Global Burden of Disease: Contribution of Inflammation. Endothelial (vascular) dysfunction is an early correlate for coronary artery disease in humans [1] and occurs in many low-grade inflammatory diseases, including rheumatoid arthritis $[2,3]$, psoriasis [4], and type 2 diabetes [5] and thereby accelerates atherosclerosis and causes cardiovascular mortality. Endothelial dysfunction is also found in severe inflammatory conditions, such as lipopolysaccharide- (LPS-) induced septic shock [1]. The PROVE IT-TIMI 22 study demonstrated that high CRP levels in patients with acute coronary syndrome predicted death from myocardial infarction [6], which adds further support to the striking correlation found between inflammation and increased cardiovascular disease risk (reviewed in [7-9]) and also provides strong evidence for a close interaction between inflammation, oxidative 
stress, and redox signaling, presenting a rich field for future investigation. Inflammation plays also a central role in neurodegenerative processes such as Alzheimer's disease [10-12] and Parkinson's disease [13, 14]. Accordingly, inflammatory disorders represent a major challenge for health care systems and societies worldwide.

The Global Burden of Disease Study (GBD) from 2012 illustrates a significant shift in the factors impacting life expectancy from communicable childhood disease toward noncommunicable diseases common in the aged $[15,16]$. This study also found that collectively, hypertension, ischemic heart disease, smoking, and cerebrovascular disease accounted for more than $50 \%$ of global deaths. These diseases and risk factors are also the leading causes of premature mortality and responsible for more than $20 \%$ of life years lost due to severe disability $[15,16]$. According to a more recent update of the GBD, diabetes and related metabolic diseases are experiencing a continuous increase in prevalence as well as incidence. Diabetes (high fasting blood glucose), hyperlipidemia (high total cholesterol), and obesity (high body mass index) rank on positions 3, 4, and 7, respectively, on the list of leading health risk factors [17]. The fact that 4 risk factors related to cerebro/cardiovascular health problems and 3 cardiometabolic risk factors were identified among the leading causes of global mortality underlines the importance of adequate cardiovascular therapy. Although inflammatory processes are not explicitly mentioned in the Global Burden of Disease Study (only lower respiratory infections are listed among the 14 most important risk factors for global deaths $[15,16])$, the contribution of dysregulated immune responses and chronic (low-grade) inflammation to atherosclerosis and subsequently cardiovascular disease development and progression is well accepted and documented (see the hazard ratio for markers of inflammation/atherothrombosis such as MMP-9 and sCD40L, autoimmune antibodies, and cardiovascular risk in Figures 1(a) and 1(b)) [18-20]. In the following two sections, two conditions with a low-grade inflammatory phenotype and increasing prevalence will be discussed in detail-aging and diabetes.

1.2. Increased Prevalence of Cardiovascular Disease at Higher Age. The number of people older than 65 years is projected to dramatically increase in the next decades, presenting demographic change as an emergent issue in Western society [21]. Demographic shifts toward an older population will have economic effects like increased spending on retirement funds and will also amplify the economic burden for healthcare costs. The expected increase in cardiovascular disease (CVD) burden corresponding to the shift toward an older population creates an urgent need for research in the field to supplement limited existing knowledge in order to reduce the predicted increase in morbidity and mortality. In particular, it is necessary to elucidate the pathophysiological convergence of separate diseases with comorbidities that also increase as age advances [22-24]. CVD is a leading contributor to morbidity and mortality in the elderly and closely correlated with age [25] due to the phenomenon of "vascular aging," which encompasses all age-associated changes in vessels. Since aged vessels are more prone to atherosclerotic lesions, vascular injury, impaired angiogenesis, and calcification [26], it is clear that the aging endothelium is more and more unable to regulate all its tasks, manifesting as significant impairment of endothelium-dependent relaxation (endothelial dysfunction) in elderly people [27, 28]. This supports the demographic data illustrating a positive correlation between CVD incidence or complications and age, also supported by higher use of CVD medications with increasing age (Figure 1(c)) [29]. Mechanistically, both smooth muscle and endothelial cells are involved in the vascular aging process because aging leads to arterial stiffness and endothelial dysfunction, both known to correlate with future cardiovascular events in humans [30].

Since endothelial dysfunction promotes thrombocyte activation [31], vasoconstriction, leukocyte activation/infiltration [32, 33], and smooth muscle cell proliferation (intima-media thickness) [34] in the vessel wall, it is consequently an early predictor for the development of atherosclerosis, hypertension, and future cardiovascular events (for review, see [9]). Recently, three interdependent key players in the vascular aging process have been identified that can trigger endothelial dysfunction: impaired nitric oxide signaling, oxidative stress, and inflammation [35]. Age-dependent endothelial dysfunction and oxidative stress are found in all vessels, from macrovessels like the aorta and coronary arteries to resistance vessels of the microcirculatory system (for review, see [36]). Paralleling the wide-ranging presence of oxidative stress, endothelial dysfunction in the elderly is not only associated with CVD but also with other disorders related to aging, such as erectile dysfunction, renal dysfunction, Alzheimer's disease, or retinopathy [37-40]. Highlighting this, studies by Mayhan et al. demonstrated an impaired eNOS-dependent reactivity in cerebral arterioles, which was additionally positively associated with increased oxidative stress at higher age [41]. Endothelial dysfunction has been demonstrated in aging retinal vessels [42] and also implicated as a contributor in neurodegenerative disease [43, 44]. Impaired NO signaling, vascular inflammation, and oxidative stress are key players in the pathogenesis of agedependent endothelial dysfunction, as demonstrated by our group and many others (for review, see $[45,46]$ ). In combination, these data all implicate aging as an independent risk factor for cardiovascular disease, mainly due to oxidative stress-induced endothelial dysfunction and low-grade inflammation $[47,48]$.

Plasma concentrations of some inflammatory markers (sVCAM-1, IL-6, MCP-1, and others) are positively correlated with age, independent of other cardiovascular risk factors [49]. In both men and women, older age was associated with higher levels of interleukin-6 (IL-6), IL-1 receptor antagonist (IL-1ra), IL-18, C-reactive protein (CRP), and fibrinogen (most changes persisted after correction for other risk factors), while soluble IL-6 receptor (sIL-6r) increased significantly with age only in men (Figure 1(d)) [50]. A meta-analysis (32 cross-sectional studies, $n>23,000$ subjects) also revealed that age-associated frailty and prefrailty are associated with higher inflammatory parameters, in particular CRP and IL-6 [51]. Hazard ratios for serum CRP levels as well as IL- 6 at baseline and incident 


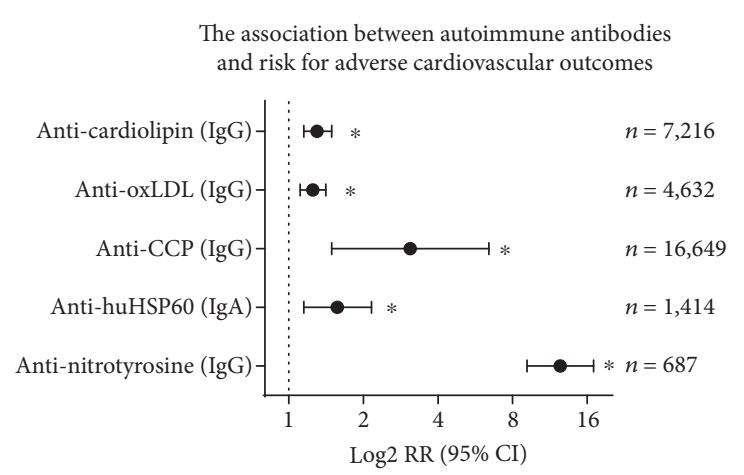

(a)

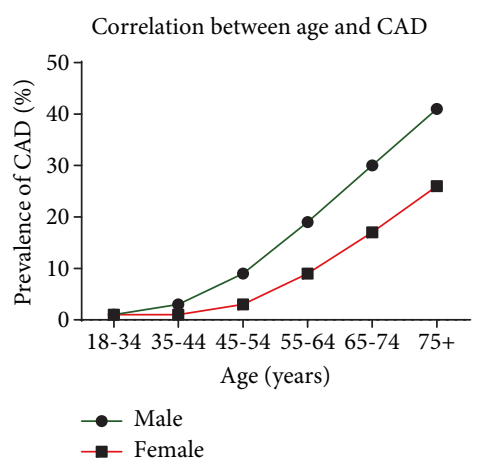

(c)

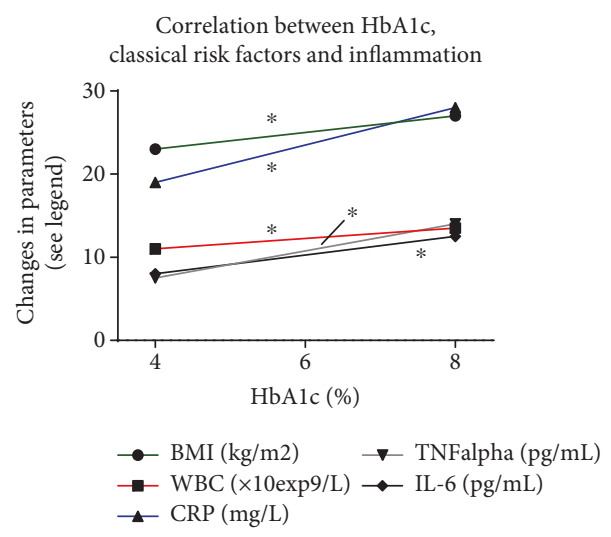

(e)
The association between inflammation markers and coronary heart disease mortality (meta-analysis)

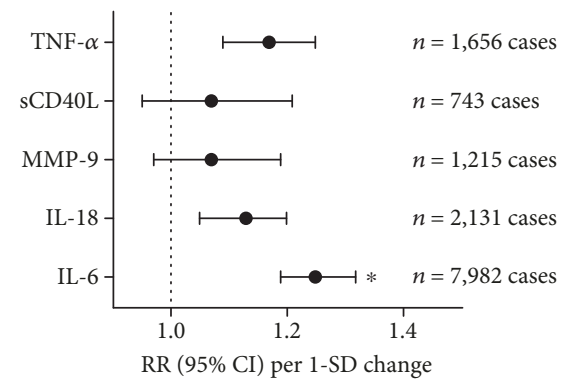

(b)

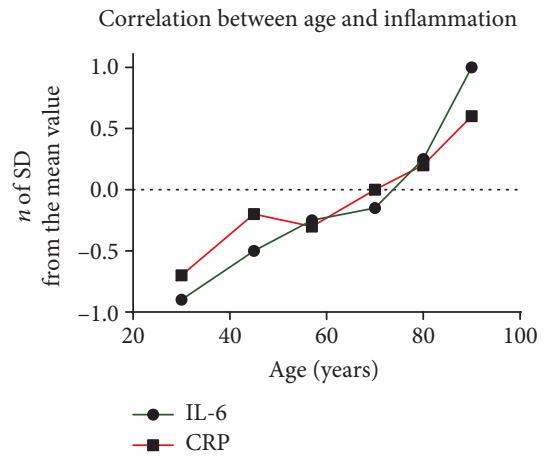

(d)

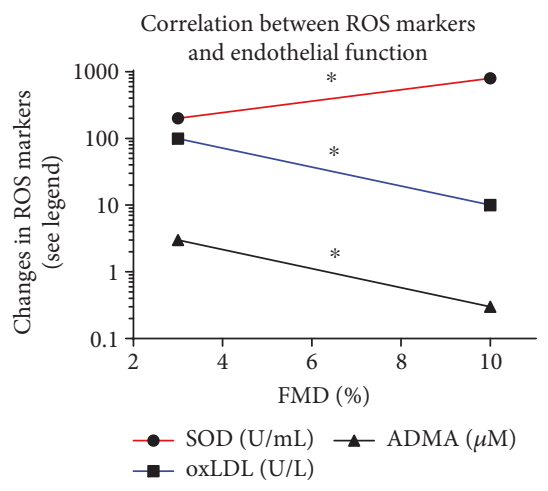

(f)

FIGURE 1: Impact of autoimmune antibodies and inflammation markers on cardiovascular events or mortality-associations between age or glycemic state and inflammation. (a) Hazard ratios for adverse cardiovascular outcomes in correlation with autoimmune antibodies (IgG subtype) obtained by meta-analysis and adjustment for age, sex, smoking status, adiposity markers, blood pressure, and/or lipid markers (number of cases as indicated) and adjustment for classical confounders. $*$ indicates significant differences to the control group. oxLDL $=$ oxidized low-density lipoprotein; CCP = cyclic citrullinated protein; HSP60 = heat shock protein 60 . Graph was generated from tabular data by Thomson et al. [284] for anti-nitrotyrosine or Iseme et al. [18] for all other autoimmune antibodies. (b) Hazard ratios for all coronary heart disease mortality in correlation with markers of inflammation interleukin- (IL-) 6, IL-18, matrix metalloproteinase- (MMP-) 9, soluble CD40 ligand (sCD40L or CD154), and tumor necrosis factor- (TNF-) $\alpha$ obtained by meta-analysis and adjustment for age, sex, smoking status, adiposity markers, blood pressure, and/or lipid markers (number of cases as indicated). Risk increases are shown per 1 SD changes of cytokines. * indicates significant differences to the control group. Redrawn from tabular data by Kaptoge et al. [19]. (c) Prevalence of coronary artery diseases (CAD) increases with the progressing age and gender in the general German population. Drawn from results of the DETECT study [29]. (d) Mean values of inflammatory markers (CRP = C-reactive protein and IL-6) according to the age group expressed as number of standard deviations from the sex-specific population mean to make them independent of different units of measure. Only data for men $(n=595)$ are shown, but those for women $(n=748)$ look very similar. Other markers of inflammation show similar correlations (e.g., IL-18 and fibrinogen). Graph was roughly estimated from tabular data by Ferrucci et al. [50]. (e) The correlation between HbAlc and potential risk factors. The correlation between HbAlc and body mass index (BMI), white blood cells (WBC), CRP, TNF- $\alpha$, and IL-6 ( $n=221$ subjects). Graph was roughly estimated from graphical data by Wang et al. [62]. (f) The correlation between serum superoxide dismutase (SOD) activity, oxLDL and asymmetric dimethylarginine (ADMA) levels, and endothelial function measured by FMD ( $n=59$ healthy and chronic kidney disease subjects). Graph was roughly estimated from graphical data by Yilmaz et al. [117]. * indicates significant differences to the control group. 
of age-associated frailty were 1.06 (95\% confidence interval (CI): 0.78-1.44) and 1.19 (95\% confidence interval (CI): $0.87-1.62$ ), respectively, after adjustment for 9 potential confounders [51].

1.3. Increased Prevalence of Cardiovascular Disease in the Setting of Diabetes and Related Cardiometabolic Disease. A recent estimation by the World Health Organization (WHO) from October 2018 states that "the number of people with diabetes has risen from 108 million in 1980 to 422 million in 2014," with a more rapid increase in middle- and lowincome countries [52]. WHO further states that "in 2016, an estimated 1.6 million deaths worldwide were directly caused by diabetes and another 2.2 million deaths were attributable to high blood glucose in 2012." This figure is only expected to rise dramatically within the next decades. In addition to being a leading cause of mortality (see recent GBD data [17]), diabetes is the most common cause of blindness, nontraumatic amputations, and end-stage renal disease in adults. The cost associated with this disease is devastating. Compounding the costs of diabetes and heart disease paints a bleaker picture; in China from 2006-2015, a $\$ 558$ billion loss in national income was attributable to heart disease, stroke, and diabetes. Diabetes mellitus is an accepted independent risk factor in the development of coronary heart disease with a notable adverse effect on survival; in diabetics, the risk for death from coronary artery disease is 2- to 4 -fold higher [53]. Demographic statistics underline the role of diabetes in exacerbating coronary disease. In Germany, a diabetic suffers a heart attack every 19 minutes. Additionally, the risk of myocardial infarction (MI) for diabetics diagnosed within 10 years without a preceding $\mathrm{MI}$ is equal to the risk level of nondiabetics with a previous MI [53]. 24-hour mortality is also considerably higher following MI surgery in diabetic patients (14\%) as compared to nondiabetic patients (5\%), according to the "Münchner" MI database [54, 55]. Also, the numbers of end-stage renal disease are dramatically higher in patients with diabetes, also with a clear correlation with age [56].

The upward trend in diabetic prevalence is exemplified in the recent update to the GBD, which shows that diabetes and related metabolic diseases have increased in prevalence and incidence between 1990 and 2015 [17]. Diabetes, defined as high fasting blood glucose, moved from rank 7 to 3 in the list of global health risk factors. Diabetes-associated risk factors also moved up in rank, hyperlipidemia from rank 9 to 6 , and obesity from rank 7 to 4 [17]. The increasing prevalence of these risk factors demonstrates the impact of modern lifestyle changes (e.g., unhealthy diet, physical inactivity, and probably environmental triggers) in both Western and industrializing societies. Hyperlipidemia and diabetes are both classical cardiovascular risk factors that are connected through an underlying association with endothelial dysfunction $[57,58]$. In addition, they are often both present alongside arterial hypertension, a strong trigger for endothelial dysfunction [59]. A meta-analysis revealed a hazard ratio for cardiovascular mortality for screen-detected diabetes of 3.42 (95\% confidence interval (CI): 2.23-5.23) [60]. In a population-based study $(n=631)$, T2DM was crosssectionally associated with both endothelial dysfunction and low-grade inflammation explaining approximately $43 \%$ of the increase in cardiovascular mortality risk in the diabetic group [61]. Markers of inflammation (CRP, IL-6, and TNF- $\alpha$ ), white blood cell count (as a general parameter of the immune system state), and body mass index (surrogate for the risk factor obesity) show a positive correlation with increasing $\mathrm{HbAlc}$ values (Figure 1(e)) [62]. Vice versa, increased markers of inflammation are associated with higher risk for type 2 diabetes development [63]. Hazard ratios for serum CRP levels as well as IL-6 at baseline and risk of T2DM in male and female population were 1.26 (95\% confidence interval (CI): 1.16-1.37) and 1.31 (95\% confidence interval (CI): 1.17-1.46), respectively (22 cross-sectional studies, $n=40,735$ subjects for CRP; 10 cross-sectional studies, $n=19,709$ subjects for IL-6) [63]. Of note, in diabetic and nondiabetic patients with symptomatic chronic heart failure, the $\mathrm{HbA} 1 \mathrm{c}$ level is an independent progressive risk factor for cardiovascular death, hospitalization for heart failure, and total mortality [64]. The hazard ratios per $1 \%$ higher HbAlc levels (adjusted for age and sex) of the primary composite outcome (CV death or HF hospitalization), cardiovascular (CV) death, hospitalization for worsening heart failure (HF), or death from all causes were 1.25 (95\% confidence interval (CI): 1.20-1.31), 1.24 (95\% confidence interval (CI): 1.17-1.31), 1.25 (95\% confidence interval (CI): 1.19-1.31), and 1.22 (95\% confidence interval (C): 1.16-1.29), respectively $(n=2,412)$ [64]. Cardiovascular complications in diabetes mellitus are associated with oxidative stress [65, 66], as are hypercholesterolemia and arteriosclerosis $[67,68]$.

\section{The Molecular Triggers of Cardiovascular Disease}

2.1. Glucotoxicity, AGE/RAGE Signaling, RAAS Activation, and Vasoconstrictors. The mechanism by which diabetes causes cardiovascular complications is complex, but damage to the vascular endothelium is a clear contributor [69]. Proteins in the plasma and cell membrane are altered by chronic exposure to hyperglycemia through the process of nonenzymatic glycosylation, leading to the attachment of glucose molecules. Advanced glycation end-products (AGEs) are formed [70] which then proceed to inactivate nitric oxide (NO), leading to impairment of endothelium-dependent vasodilation [71]. Studies in diabetic rats have found increased AGE/RAGE signaling [72], impairment of NO/cGMP signaling [73], and also an association with NADPH oxidase-induced oxidative stress and vascular complications [74]. Some of the vascular dysfunction is attributable to a vital crosstalk between oxidative stress and AGE/RAGE components [74-76] leading to increased inflammation [77-79]. In accordance with this concept, the normalization of mitochondrial ROS formation prevented hyperglycemic damage by decreasing AGE/RAGE signaling [76]. Further support comes from observations that macrophages from gp91phox-deficient mice showed reduced responses to AGE stimulation providing a direct link between AGE/RAGE signaling and NADPH oxidase expression/activity [74]. In the same study, a connection between AGE/RAGE signaling and inflammation via increased 
VCAM-1 and tissue factor expression was demonstrated upon treatment of human endothelial cells with AGE. Furthermore, in the STZ model of diabetes in rats, increased expression of TNF- $\alpha$, IFN- $\gamma$, and ROS-producing enzymes was normalized upon multiple antioxidant therapy [80], further strengthening the connection between diabetes-induced vascular damage and inflammation. However, the disturbed vascular responses to short-term hyperglycemia are comparable to those seen in the long term, suggesting that AGE/RAGE signaling is not the only mechanism [81]. Aside from $\mathrm{AGE}$ and diacylglycerol (DAG) formation, increased oxidative stress has been observed in models of diabetes. In particular, superoxide $\left(\mathrm{O}_{2}{ }^{--}\right)$is known to contribute to the inhibition of eNOS through depletion of a critical cofactor, leading to uncoupling (reviewed in [82]). Several other pathways are also thought to contribute to the impaired vascular function and thereby increased cardiovascular risk associated with diabetes: activation of protein kinase $C$, increased metabolization of glucose via hexosamine metabolism leading to increased transcription of genes of inflammatory cytokines and PAI-1, and increased metabolization of glucose to sorbitol by the polyol pathway [83].

Activation of the renin-angiotensin-aldosterone system (RAAS) is also present in the setting of diabetes, as evidenced by higher production of DAG as well as vasoconstrictive compounds such as endothelin-1 and angiotensin-II [84]. Importantly, the activation of RAAS is also closely related to endothelial dysfunction $[85,86]$ and DAG is a potent activator of protein kinase $C[87,88]$, which leads to subsequent activation of NADPH oxidase [89]. This RAAS-induced production of superoxide was demonstrated in both clinical [90] and translational studies [91] and is further supported by evidence that suppression of RAAS prevents the progression of T2DM in hypertensive patients [92]. Thrombocytes from diabetics are larger in size, express more glycoprotein receptors on the surface, and are more prone to aggregation in response to a variety of stimuli (for review, see [93]). Another factor for consideration in the vascular consequences of diabetes is the impaired fibrinolytic activity of the endothelium in this setting [94].

2.2. Oxidative Stress as a Molecular Trigger of Cardiovascular Disease. Ohara et al. and Harrison and Ohara were the first to describe the role of oxidative stress in the development and progression of cardiovascular disease using an experimental model of hypercholesterolemia [95, 96]. In the intervening years, there has been recognition that the majority of cardiovascular diseases can be characterized as having an imbalance between the formation of ROS and ROS-degrading antioxidant systems $[67,97]$. This imbalance leads to the accumulation of superoxide, hydrogen peroxide, and other products such as peroxynitrite and hypochlorous acid and a deviation from the steady state [98]. We have previously reviewed the molecular mechanisms of endothelial (vascular) dysfunction in detail, including the involved sources of ROS and their targets, the "redox switches" in the NO/cGMP pathway, and related signaling cascades $[77,99,100]$. There are also activation pathways between different ROS sources in a crosstalk fashion [101-103], which was mainly demonstrated for the
NOX-2/mitochondrial axis [104, 105], eNOS uncoupling, and xanthine dehydrogenase/oxidase conversion [77, 78], where "ROS-induced ROS formation" can be found as originally reported for self-triggered mitochondrial ROS amplification [106]. More recently, this redox crosstalk concept was extended; in AT-II-induced hypertension, NOX-2 activation triggers Sirt3 S-glutathionylation leading to acetylation of vascular SOD2 and reduced SOD2 activity, all of which resulted in elevated mitochondrial superoxide, diminished endothelial nitric oxide bioavailability, and aggravation of hypertension $[107,108]$. This concept was even shown to be operative in tobacco smoke-induced vascular oxidative stress, endothelial dysfunction, and hypertension [109]. In short, there is an indispensable role played by oxidative stress in the development of cardiovascular disease, both mediated somewhat by the uncoupling of endothelial nitric oxide synthase (eNOS) [77], making eNOS uncoupling a hallmark of most cardiovascular diseases $[110,111]$. The functional manifestation of eNOS uncoupling is endothelial dysfunction, measurable via acetylcholine-dependent or flow-mediated dilation (FMD), making endothelial dysfunction an important, measurable, and early predictor for cardiovascular events $[68,112]$.

Because of the near-ubiquitous presence of oxidative stress in cardiovascular disease, an important link has been forged between oxidative stress and cardiovascular prognosis, supported by data from large clinical trials. A study of 636 individuals showed a positive correlation between levels of glutathione peroxidase-1, an antioxidant enzyme, and cardiovascular event-free survival [113]. Another study found that oxidative serum stress markers D-ROM (derivatives of reactive oxygen metabolites, indicative of ROS levels) and total thiol levels (representative of the total redox state) were strongly and independently associated with all-cause and CVD mortality in 10,622 men [114]. Lastly, a meta-analysis of 14 clinical trials spanning more than 1,900 participants yielded evidence that levels of 8-hydroxy-2-deoxyguanosine (a marker of oxidative DNA damage) were increased in patients with cardiovascular disease [115]. Additionally, there have been a number of small cohort clinical studies. These include evaluation of the differential effects of vitamin $\mathrm{C}$ on the flow-mediated dilation (FMD) in patients with high or low ROS burden [68]. Other studies utilizing FMD demonstrate significantly impaired response and lower levels of reduced circulating glutathione in smokers $(n=52)$ versus controls [116], a positive correlation between FMD and superoxide dismutase activity (a surrogate of antioxidant defense state), and negative correlations between FMD and oxidized low-density lipoprotein (oxLDL, oxidative stress marker and initiator of atherosclerosis) as well as asymmetric dimethylarginine (ADMA, inhibitor of eNOS and marker for cardiovascular risk) levels in 59 patients with chronic kidney disease (Figure 1(f)) [117]. In sleep apnoea, negative correlations between reactive hyperemia index (a measurement of vascular function) and malondialdehyde/8-oxo-deoxyguanosine levels were found in a study of 69 patients versus controls [118].

Large clinical trials for nonselective antioxidant therapy (mainly vitamin $\mathrm{C}$ and $\mathrm{E}$, chronic high dose oral 
administration) have been conducted and have somewhat surprisingly failed to show any health benefit for the treatment of cardiovascular disease $[119,120]$, with only few exceptions such as the European Prospective Investigation into Cancer- (EPIC-) Norfolk study with measurement of vitamin C plasma levels of all participants [121] and smallcohort studies using acute administration (mainly infusion) of vitamins (reviewed in $[8,9]$ ). Also, the synthetic antioxidant NXY-059 failed to prove benefits in stroke patients upon costly development and clinical studies [122]. Therefore, the focus has shifted to site- and source-specific antioxidant therapy (e.g., Nrf2 agonists, myeloperoxidase inhibitors, and monoamine oxidase inhibitors) through which we may glean better understanding of these antioxidant systems [120, 123-125].

The free radical theory of aging was first expressed by Harman as early as 1954 [126]. It was founded on the basis that as aging was a universal phenomenon, so must be the contributing factors that drive the process. At first, the focus of his attention was on the hydroxyl radical and molecular oxygen [127]. The theory was then extended to include mitochondria, which are now known to be the most abundant cellular source of ROS with good evidence that they are a major source of ROS in aging tissues [128, 129]. Mitochondrial DNA damage accumulates in the aging cell leading to mitochondrial dysfunction [130] and aging-related cardiovascular and neurodegenerative diseases $[35,131]$. The current iteration of the free radical theory of aging is superoxide which accumulates in an age-dependent manner and rapidly consumes NO, leading to a reduction in the availability in the endothelium and subsequent impaired vasorelaxation $[47,132]$. Deletion and/or overexpression of a single antioxidant enzyme $\left(\mathrm{SOD}^{+/-}\right.$or $\mathrm{SOD} 2^{\mathrm{tg}}, \mathrm{GPx}^{-1^{-/}}, \mathrm{GPx}-4^{-/-}$or $\mathrm{MrrA}^{-1-}, \mathrm{SOD}^{\mathrm{tg}}$, and catalase ${ }^{\mathrm{tg}}$ ) have not yielded a definitive association with shortened or lengthened lifespan [133], with the exception of mice completely deficient in SOD2, which typically do not live to term $[134,135]$. Another exception lies in the overexpression of Trx1, which increased lifespan and stress resistance [136]. Double gene deletion combinations of SOD1 alongside another antioxidant gene resulted in reduced life expectancy of mice [133, 137]. In summary, there is substantial evidence for a contribution of oxidative stress to shortening of healthspan [138-141] and to detrimental effects on physiological organ function, vascular phenotype, and inflammation $[142,143]$.

Endothelial cells are activated in response to hyperglycemia, leading to increased expression of endothelial proteins and generation of proinflammatory metabolites. This proinflammatory phenotype is conducive to adhesion of leukocytes and monocytes to the endothelium. Activation of endothelial cells in diabetes may result in a decreased NO bioavailability, which precedes disturbance in endothelial function and the decrease in coronary flow reserve. Cellular activation of the diabetic endothelium may be a key event conferring the characteristic decreased plaque stability and rupture associated with thrombus formation and vessel occlusion [144]. High plasma glucose levels increase the formation of electron-transferring compounds such as NADPH leading to increased formation of ROS. This in turn will lead to oxidative protein modifications such as 3-nitrotyrosine formation [145], oxidative disruption of the zinc-sulphur cluster and uncoupling of eNOS [146] as well as increased levels of toxic aldehydes [147], all common features of diabetes. Additionally, the increased NADPH oxidase-derived ROS leads to oxidative depletion of tetrahydrobiopterin $\left(\mathrm{BH}_{4}\right)$, a critical prerequisite for eNOS uncoupling. Importantly, eNOS uncoupling is a mediator of endothelial dysfunction, which in turn is a hallmark in the early stage of diabetes mellitus [148]. However, through overexpression of the $\mathrm{BH}_{4}$ synthesizing enzyme GTP-cyclohydrolase, $\mathrm{BH}_{4}$ levels can be rescued and eNOS uncoupling prevented in diabetic mice [149]. In diabetic patients, acute infusion of $\mathrm{BH}_{4}$ recoupled eNOS and improved endothelial function [148]. Supplementation with lipoic acid or vitamin $\mathrm{C}$ also improved endothelial function in diabetic subjects [150]. Complementary to this, antioxidants have been shown to have a beneficial effect on endothelial function [147, 150-152].

2.3. Inflammation as a Molecular Trigger of Cardiovascular Disease. Inflammation is an important player in both chronic disease and cardiovascular disease. Chronic kidney disease, nonalcoholic fatty liver disease, and neurodegeneration are all either triggered by or associated with low-grade inflammation $[8,9,101]$. Low-grade inflammation is also a contributor to the hallmark oxidative stress associated with most cardiovascular disease [7, 100, 153], representing a cardiovascular risk factor [19] that can be targeted by pharmacological treatment [154]. Systemic lupus erythematosus, rheumatoid arthritis, and psoriasis are autoimmune diseases that bridge the gap between chronic and cardiovascular disease, as they possess increased cardiovascular risk [155-158]. Furthermore, psoriasis has even been characterized as an independent risk factor for cardiovascular disease [4] where patients presenting with psoriasis or associated inflammatory arthritis are recommended by the European League Against Rheumatism to undergo cardiovascular therapy [159]. In line with this, systolic blood pressure of patients with rheumatoid arthritis or psoriasis was reduced by immune-suppressive therapy [160]. The basis in this recommendation lies in the presentation of endothelial dysfunction found in psoriatic patients as well as those with rheumatoid arthritis $[161,162]$. Since recent research has revealed links between inflammatory signaling networks and the brain, autonomic nervous system, and spleen in both atherosclerosis and myocardial infarction [163], it is not surprising that additional studies found that targeted anti-inflammatory therapy lowered cardiovascular mortality in psoriasis (IL-17/IL-23 axis) [164-166], systemic lupus erythematosus (IL-17A signaling) [167], and rheumatoid arthritis (IL-6, TNF- $\alpha$, and IL-17A cascades) $[168,169]$. Several of the aforementioned cytokines (e.g., interleukins IL-6, IL-1 $\beta$, IL-17A, and tumor necrosis factor alpha (TNF- $\alpha$ )) are positively correlated with cardiovascular events [7]. Accordingly, clinical trials of IL-1 receptor blockers or antagonists (anakinra, rilonacept, canakinumab (CANTOS trial), and gevokizumab) in patients with cardiovascular disease are underway or already completed [170]. Also, recent discoveries of Genome-Wide 
Association Study (GWAS) on risk loci in a large population cohort ( $n=194,427$ participants) suggest an important role of arterial wall-specific inflammation processes in the development and progression of coronary artery disease; for example, SNPs in or near genes involved in cellular adhesion, leukocyte migration and atherosclerosis (PECAM1, rs1867624), and coagulation and inflammation (PROCR, rs867186 (p.Ser219Gly)) were identified [171]. Also, other recent GWAS data contained several risk loci that are linked to cardiovascular inflammation [172]. Endothelial microparticles (EMPs) are vesicles with a size range of $0.1-1.0 \mu \mathrm{m}$ that are released upon endothelial cell activation or apoptosis and promote atherosclerosis by the induction of oxidative stress and vascular inflammation [173]. Therefore, the pleiotropic anti-inflammatory effects of the most successful cardiovascular drugs (e.g., statins, ACE inhibitors, and AT1R blockers) as well as new antidiabetic drugs (gliptins, GLP-1 analogues, and SGLT2 inhibitors), which have been found to possess potent immune-suppressant properties in experimental analyses and postmarket launch, represent valuable therapeutic add-ons $[8,174,175]$. All these data support a link between cardiovascular disease and chronic autoimmune diseases through inflammation (also reviewed in $[155,157]$ ). It is worth noting that also negative data exists as shown by the recent study on low-dose methotrexate for the prevention of atherosclerotic events ( $n=4,786$ patients) [176]. The study was stopped earlier since methotrexate did neither reduce levels of IL- $1 \beta$, IL- 6 , and CRP nor the number of cardiovascular events and most importantly caused higher incidence of non-basal-cell skin cancers as compared to placebo.

The presence of low-grade inflammation is also notable as immunosenescence within the aging process which contributes to a higher prevalence of metabolic and cardiovascular complications in the elderly [177]. The clear overlap between the risk factors of cardiovascular diseases and diabetes, particularly regarding inflammation, implies that the immunosenescence could represent a key player for agingassociated diseases and increased comorbidity in the elderly [22-24]. Therefore, normalizing the chronic inflammatory phenotype and additionally, targeting mitochondrial oxidative stress (mtROS) could represent a promising strategy to increase healthspan [178]. mtROS formation increases with advancing age [45] and leads to the activation of immune cells and their phagocytic NADPH oxidase, thereby stimulating cytokine release and the inflammasome [78, 179-183]. The hypothesis that targeting mitochondrial ROS could be a strategy for suppression of the chronic inflammatory phenotype of advancing age was recently supported by a study in an animal model for metformin-dependent AMPactivated protein kinase activation. Elderly animals had an increased lifespan and healthspan as a result of suppression of inflammation and oxidative damage [184]. As highlighted in the preceding sections, there is also a vital crosstalk between oxidative stress and AGE/RAGE components [74-76] leading to increased inflammation [77-79]. The crosstalk between oxidative stress and inflammation will be discussed in more detail in the subsequent section.

Also, hypertension, a leading cardiovascular risk factor for global mortality and disease burden, is now seen as an inflammatory disease [185]. The molecular mechanisms are explained in detail by experimental animal data in the following section. Of note, more recently autoimmune mechanisms were reported as the driving force of hypertension leading to infiltration of immune cells, oxidative stress, and stimulation of the intrarenal angiotensin system by the activation of the innate and adaptive immunity $[186,187]$. This new concept is mainly based on the discovery of autoantigens (e.g., isoketal-modified proteins by animal and human studies).

2.4. Interplay of Oxidative Stress and Inflammation. Inflammatory processes clearly connect vascular dysfunction and cardiovascular diseases such as arterial hypertension, hypercholesterolemia, and coronary artery disease [7, 153]. Additionally, recent data provide a basis for this tight association between redox regulatory pathways and inflammation via redox activation of immune cells by mitochondrial superoxide/hydrogen peroxide and the subsequent activation of the phagocytic NADPH oxidase (NOX-2) [78, 188]. Cultured $\mathrm{T}$ cells isolated from spleens of in vivo angiotensin-II(AT-II-) treated mice showed increased mitochondrial superoxide/hydrogen peroxide formation and thereby increased TNF- $\alpha$ production, as well as heightened energy metabolism via increased ATP production. All of these alterations were corrected by ex vivo treatment with mitoTEMPO, a mitochondria-targeted antioxidant and SOD mimetic [183]. Mitochondrial superoxide/hydrogen peroxide formation is a strong trigger (via the redox crosstalk $[77,101])$ for NOX-2 activation, which is in turn critical for the activation, recruitment, and infiltration of myelomonocytic cells $[189,190]$ and T cells [191]. Evidence for this was demonstrated by Wenzel et al. by adoptive cell transfer of monocytes in mice with deficiency of myelomonocytic cells. Myelomonocyte-deficient mice were resistant to the effects of AT-II infusion, but lost this resistance upon transfer of monocytes from wild-type mice, but not upon transfer of monocytes from gp91 ${ }^{\text {phox-/- }}\left(\right.$ Nox $\left.^{-/-}\right)$[189]. Similarly, Guzik et al. have shown that adoptive cell transfer of $\mathrm{T}$ cells (but not $\mathrm{B}$ cells) from wild-type mice but not from $\mathrm{p} 47^{\text {phox-l- }}$ mice restored hypertension and all adverse effects of AT-II infusion in RAG1 ${ }^{-1-}$ mice with deficiency in most immune cells [191]. A substantial role of B cells for the development of hypertension by AT-II treatment was also shown [192]. Conversely, the normalization of blood pressure in hypertensive humanized mice suppressed infiltration of immune cells [193], compounding evidence that inflammation is a key player in redox-regulated vascular dysfunction via redox crosstalk of mitochondria and NOX-2 in immune cells and through various other signaling events like fatty acid metabolism (e.g., formation of leukotrienes or oxLDL). More recently, a redox-dependent crosstalk between inflammatory and coagulation pathways was described for experimental hypertension that contributes to increased tissue factor levels, leukocyte-platelet interactions, vascular infiltration of immune cells, oxidative stress, endothelial dysfunction, and blood pressure, all of which were normalized by pharmacological, genetic, or immunological blockade of VCAM-1, tissue factor, thrombin, FXI, GPIb $\alpha$, and Mac-1 signaling [194]. Consideration of platelet functional state for estimation of 
vascular inflammation and cardiovascular risk may become even more attractive since the mean platelet volume shows associations with hypertension and rheumatoid arthritis [195] and clinical impact as a risk marker, although technical problems still prevent the broad clinical use of this parameter [196]. Details on the interaction of immune cells (mainly monocytes/macrophages), platelets, and vascular cells with consideration of redox-based mechanisms can be found elsewhere [100, 197-199].

Also, protein oxidation, oxidative DNA damage and oxidized nucleotides, redox activation of protein kinases (e.g., ERK1/2, JNK), and redox inhibition of protein phosphatases regulate inflammatory processes. For example, mtROS can increase mitochondrial permeability (by opening of the mitochondrial permeability transition pore) with subsequent release of (oxidized) mtDNA, which represents a damageassociated molecular pattern (DAMP) and initiates a process called "sterile inflammation" (Figure 2) [100, 200]. Other examples for the contribution of ROS to inflammation pathways are redox-dependent formation of protein complexes (e.g., p53-JNK, Nrf2-Keap1), redox regulation of mediators of inflammation (e.g., HMGB1, S100 proteins, and DAMPs), and redox modulation of transcription factors involved in inflammatory pathways (e.g., Nrf2, AP-1, NF- $\kappa$ B, and HIF$1 \alpha$ ) [201-203]. Additional recent evidence linking oxidative stress, inflammation, and the development of hypertension lies in the formation of isoketals, highly reactive compounds that form protein adducts that can then trigger immune cell infiltration into the vasculature and high blood pressure. [204]. The cellular redox potential controls the migration of monocytes/recruitment of macrophages [205]. Finally, redox modifications of MAPK phosphatase 1 [206] and Slingshot1L-binding protein 14-3-3zeta [207] as well as changes in the S-glutathionylation pattern [208] represent other potential redox regulatory mechanisms. This crosstalk between oxidative stress and inflammation in general is supported by evidence for specific redox regulation of the cytokine release controlling NLRP3 inflammasome (Figure 2) [179-181, 209-211], the central organizer of inflammation high-mobility group box 1 (HMGB1) [203, 212-215], and the antibacterial process of neutrophil extracellular trap (NET) formation [216-221] as well as transcription factors and other essential mediators of inflammation (reviewed in [100]). In particular, the NLRP3 inflammasome and HMGB1 represent major "redox hubs" of the inflammation cascade and are regulated by redox-sensitive cysteine residues. The crystal structure of NLRP3 contains a highly conserved disulfide bond connecting the PYD domain and the nucleotidebinding site domain, which is highly sensitive to altered redox states [211]. Reduction of all thiols in HMGB1 makes it a chemokine, a disulfide bond between Cys23 and Cys45 converts it to a proinflammatory cytokine, and oxidation of all thiols makes it inactive [215].

Direct molecular proof for the interplay of oxidative stress and inflammation comes from studies of mice with genetic modulation of antioxidant defense or ROSproducing enzymes, all of which show an alteration of the inflammatory phenotype (Table 1). Our own observations of a correlation between a marker of inflammation (CD68 staining) and overall vascular ROS formation (dihydroethidine (DHE) staining) revealed an age-dependent progressive phenotype of low-grade inflammation in glutathione peroxidase-1- (GPX-1-) deficient mice [46, 143]. Since global vascular ROS formation was correlated with mitochondrial ROS formation and all other parameters were linked to global vascular ROS formation, one can assume that mitochondrial and cellular ROS formation significantly contributes to eNOS dysregulation/uncoupling, vascular function, and low-grade inflammation during the aging process [46, 143]. White blood cells from mice with heterozygous mitochondrial superoxide dismutase (MnSOD or SOD2) deficiency showed higher NOX-2 activity and endothelial dysfunction, all of which were further aggravated upon challenges with AT-II [78]. Likewise, genetic deletion of heme oxygenase-1 (HO-1) caused induction of NOX-2 at the protein level, increased levels of vascular ROS, and endothelial dysfunction, all of which were further aggravated by AT-II-induced hypertension [222]. HO-1 deficiency also caused increased expression of the C-C chemokine receptor type 2 (CCR2 or CD192), leukocyte rolling, and adhesion to the endothelial cell layer and numbers of infiltrated neutrophils and monocytes. More examples for pro- or anti-inflammatory effects by genetic modulation of antioxidant defense or ROS-producing enzymes can be found in Table 1 .

\section{Anti-Inflammatory and Antioxidant Properties of Dipeptidyl Peptidase-4 (DPP-4) Inhibitors, Glucagon-Like Peptide-1 (GLP-1) Analogs, and Sodium-Glucose Cotransporter 2 (SGLT2) Inhibitors}

Current evidence suggests that SGLT-2 inhibitors are more effective than both GLP-1 agonists and DPP-4 inhibitors in reducing the risk of hospitalization for heart failure (HHF) in T2DM (87,162 participants) [223], although the latter showed more potency in reducing HHF in T2DM than sulphonylureas (e.g., glibenclamide) and thiazolidinediones (glitazones) (127,555 patients in the meta-analysis) [224]. According to another large-scale clinical study, incretinbased therapy by GLP-1 agonists or DPP-4 inhibitors showed a trend for decreased risk of hospitalization for heart failure (1,499,650 patients, with 29,741 hospitalized for heart failure) [225]. Both drug classes have cardioprotective effects in models of myocardial infarction [226-229]. In addition, both drug classes provide potent anti-inflammatory properties [230-232], which could be beneficial in many cardiovascular but also neurodegenerative diseases. In the following two subsections, we will present and discuss our recent findings on anti-inflammatory effects of SGLT-2 inhibitors, GLP-1 agonists, and DPP-4 inhibitors in animal models of diabetes type $1 / 2$ as well as experimental sepsis.

3.1. DPP-4 Inhibition and GLP-1 Supplementation. Glucagon-like peptide-1 (GLP-1) is a peptide hormone with known anti-inflammatory properties. Endogenous GLP-1 is degraded by the exopeptidase dipeptidyl peptidase- 4 (DPP-4) within seconds under physiological conditions. It 


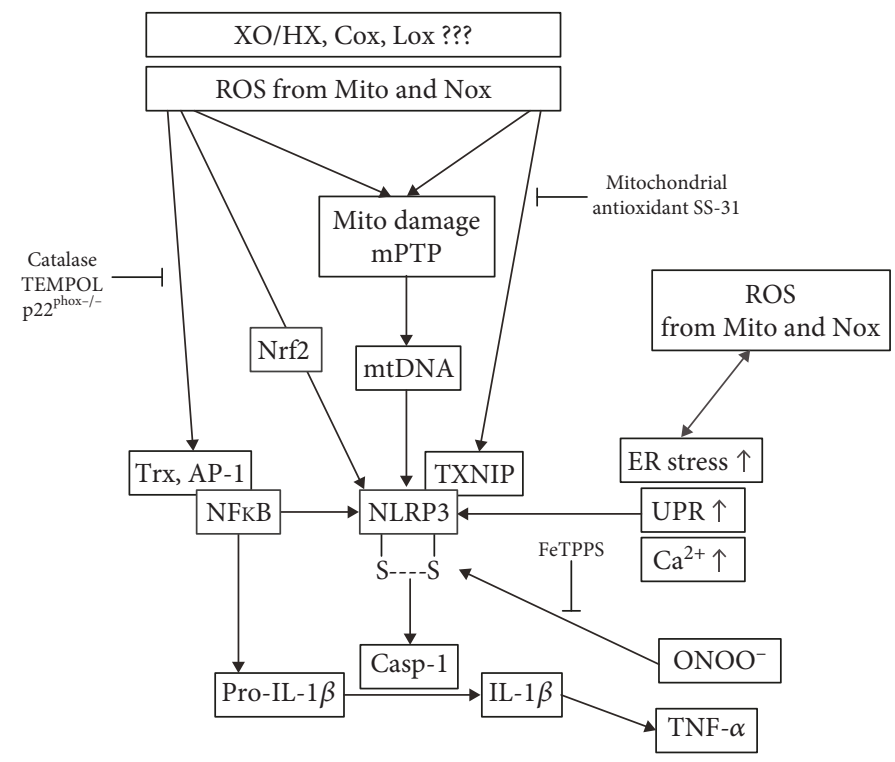

FIGURE 2: Crosstalk between oxidative stress and inflammation. Interaction between ROS and mediators of inflammation exemplified by redox regulation of the transcription factors Nrf2 and NF- $\kappa$ B and the NLRP3 inflammasome as well as their downstream targets. In several pathways, inhibitory effects by antioxidants or genetic deletion of ROS sources are indicated. Modified from Wenzel et al. [100] (with permission from Elsevier. (c) 2017 Elsevier Inc.; all rights reserved).

increases insulin release from beta-cells in the pancreas and consequently its synthetic analogs are used for treatment of type 2 diabetes. Clinical trials, like LEADER (liraglutide) and SUSTAIN-6 (semaglutide), demonstrated that GLP-1 analogs reduce the risk of cardiovascular events in T2DM patients, an effect beyond glycemic control [233, 234]. In the LEADER trial, patients suffering from T2DM were treated with standard therapy or standard therapy plus liraglutide. In this clinical trial, the risk of death from cardiovascular causes nonfatal myocardial infarction or nonfatal stroke was lower with liraglutide than with placebo (hazard ratio, $0.87 ; 95 \%$ confidence interval (CI), 0.78 to 0.97 ; $p<0.001$ for noninferiority; $p=0.01$ for superiority). According to these results, another trial (SUSTAIN-6) found similar results for the GLP-1 analog semaglutide. The primary endpoint (death from cardiovascular causes, nonfatal myocardial infarction, or nonfatal stroke) occurred in $6.6 \%$ of the study population in the semaglutide group and in $8.9 \%$ in the placebo group (hazard ratio, 0.74 ; $95 \%$ confidence interval (CI), 0.58 to $0.95 ; p<0.001$ for noninferiority) $[233,234]$. Other clinical trials investigated the effects of GLP-1 on cardiovascular disease and demonstrated cardioprotective effects after myocardial infarction [235], reduction of blood pressure [236], and an improvement of endothelial dysfunction [237]. Interestingly, it has been shown that GLP-1 levels in patients with coronary artery disease are reduced [238]. Experimental studies provide first evidence for a modulating effect of GLP-1 on inflammatory cells, and it has been recently published that the GLP-1 degrading enzyme dipeptidylpeptidase-4 (DPP-4) promotes vascular adipose tissue inflammation and insulin resistance in obesity [239], also suggesting a potential role of broader "metabolic alterations" in redox-mediated inflammation. Other studies report vascular protection by DPP-4 inhibition in animal models of atherosclerosis due to ameliorated vascular inflammation $[174,175]$. Also, all major complications of experimental nonalcoholic fatty liver disease (NAFLD) and nonalcoholic steatohepatitis (NASH), namely, hepatic and vascular inflammation, were improved by DPP-4 inhibitor therapy by linagliptin and sitagliptin [240]. The therapy with GLP-1 analogs may be also useful for patients with NAFLD or NASH [241]. However, it remains puzzling why clinical trials for DPP-4 inhibitors like saxagliptin failed to improve the cardiovascular outcome or even increased the rate of hospitalization for heart failure [242].

Sepsis is an inflammatory disease and can affect the whole organism. Depending on the individual patient's characteristics, a pneumonia can expand to a "systemic inflammatory response syndrome" (SIRS) with a mortality of $40 \%$, making it a leading cause of death in Western countries [243]. In experimental sepsis, the anti-inflammatory effects of GLP-1 analogs and DPP-4 inhibition were recently confirmed [244, 245]. In lipopolysaccharide- (LPS-) induced septic shock, the DPP-4 inhibitor linagliptin improved vascular function and reduced vascular superoxide formation [248]. Linagliptin reduced oxidative burst in whole blood and ameliorated infiltration of white blood cells to the vascular wall. Furthermore, the expression of NADPH oxidase was reduced [245]. In another study, our group demonstrated that DPP-4 inhibition by linagliptin, GLP-1 supplementation by liraglutide, and genetic deletion of DPP-4 improve survival of LPS-induced endotoxemia in mice [246]. In accordance, EPR (electron paramagnetic resonance) spectroscopy studies revealed reduced iNOS activity (nitrosyliron hemoglobin $(\mathrm{HbNO})$ ) in whole blood of endotoxemic rats under linagliptin and liraglutide therapy [246]. Likewise, mRNA expression of IL-6, iNOS, ICAM-1, MCP-1, TNF- $\alpha$, and VCAM-1 in aortic tissue of endotoxemic rats was 


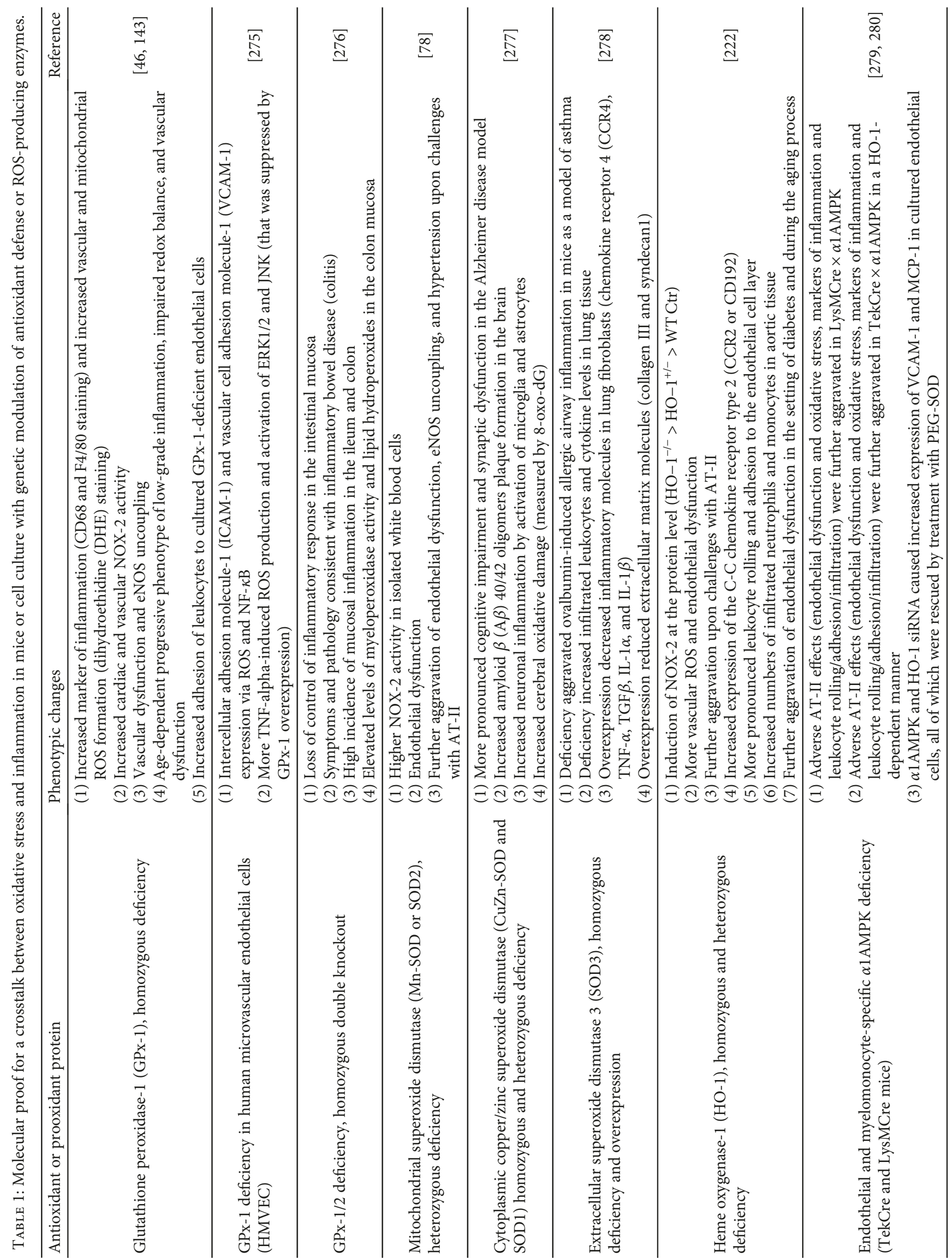




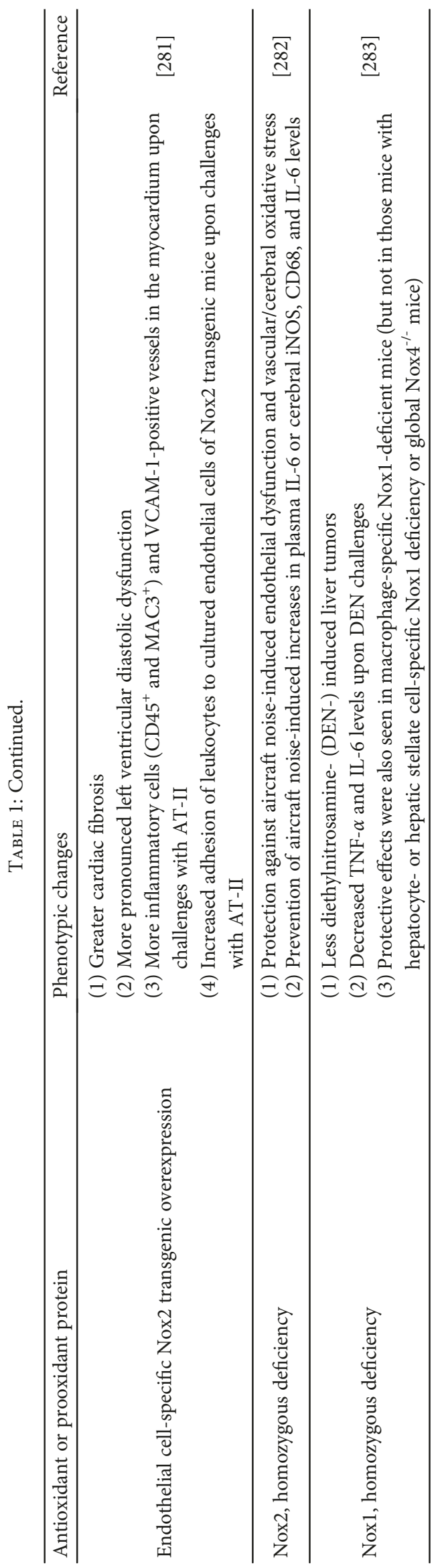




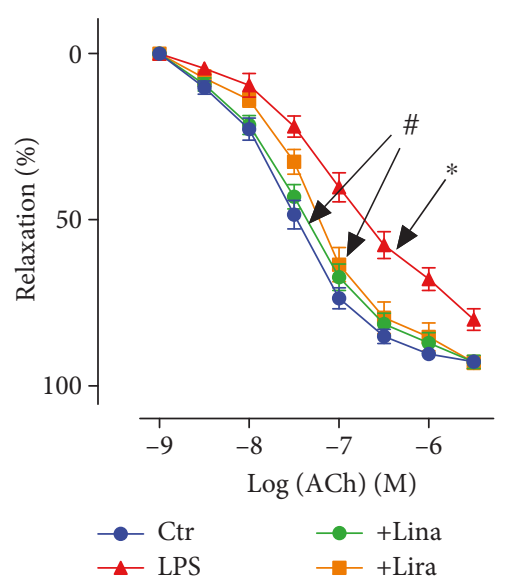

(a)

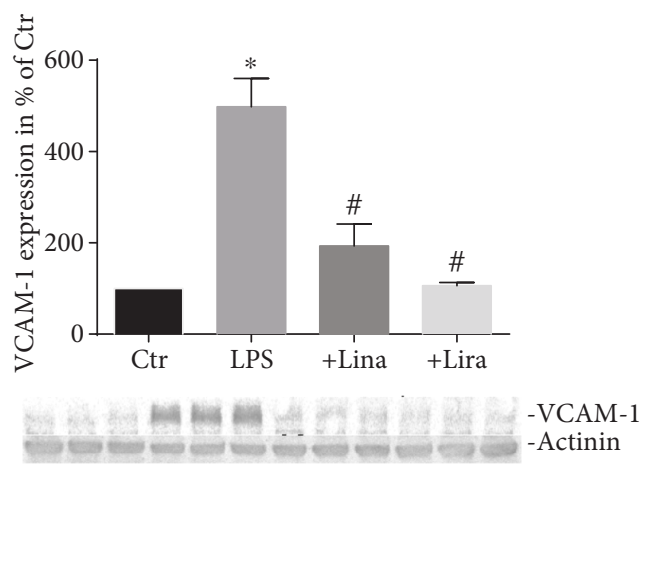

(b)

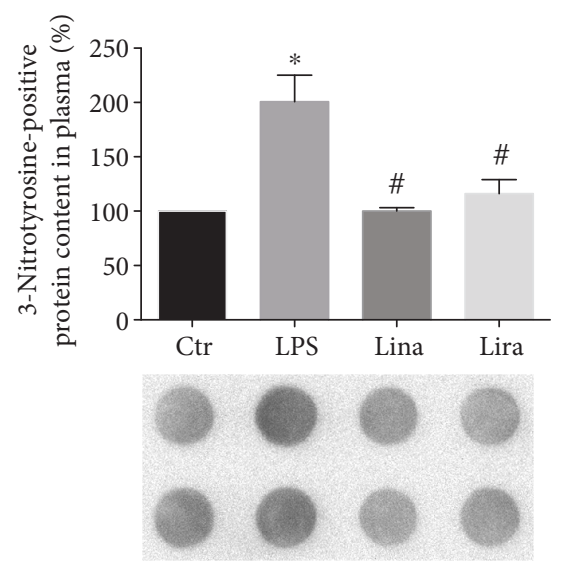

(c)

FIGURE 3: Antioxidant, anti-inflammatory, and vasculoprotective effects by the DPP-4 inhibitor or GLP-1 analog therapy in septic rats. (a) Endothelium-dependent ( $\mathrm{ACh}$ ) relaxation was determined by isometric tension studies in rat aortic ring segments. (b) Aortic expression of inflammatory protein VCAM-1 was assessed by Western blotting analysis and specific antibodies. (c) Levels of 3-NT-positive proteins in plasma were assessed by dot blot analysis and specific antibodies. Representative blots are shown below the densitometric quantification. The data are mean \pm SEM from $4-8\left(\mathrm{a}-\mathrm{c}\right.$ ) rats/group. ${ }^{*} p<0.05$ vs. the control and ${ }^{\#} p<0.05$ vs. LPS. Adopted from Steven et al. [246] (with permission from Springer-Verlag Berlin Heidelberg. Copyright (c) 2015, Springer).

suppressed by both drugs, an observation that was accompanied by improved endothelial function and reduced nitrooxidative stress as measured by 3-nitrotyrosine-positive proteins (Figures 3(a)-3(c)) [246]. Importantly, these protective effects are beyond glycemic control since no changes in blood glucose levels were reported.

Endotoxemic mice suffer from severe thrombocytopenia, which appears in the setting of disseminated intravascular coagulation. Microvascular thrombosis in the pulmonary circulation (detected by fluorescence imaging) led to severe organ damage and increased lactate dehydrogenase (LDH) activity. Vascular function was impaired, and increased markers of inflammation in the aorta and whole blood (leukocyte-dependent whole blood oxidative burst, $\mathrm{HbNO}$ ) were present (Figure 4(a)) [247]. Treatment with linagliptin (DPP-4 inhibitor) and liraglutide (GLP-1 analogue) as well as genetic deficiency of DPP-4 (DPP- $4^{-/-}$mice) improved all these parameters. Interestingly, in GLP-1 receptor knockout mice, liraglutide completely and linagliptin partially lost their therapeutic efficiency as demonstrated by survival studies (Figure 4(b)) [247]. Incubation of cultured monocytes with GLP-1 analogues inhibited the monocytic oxidative burst by the elevation of cAMP and increased protein kinase A (PKA) activation as a potential mechanistic explanation of the beneficial GLP-1-dependent signaling effects.

Besides inflammatory cells, inhibition of DPP-4 and GLP-1 might also have impact on thrombocytes. The experiments in endotoxemic animals revealed that the bleeding time was ameliorated by DPP-4 inhibition and GLP-1 supplementation in rats, a first evidence for a role of DPP-4/GLP-1 signaling in hemostasis [246]. Antiaggregatory effects by sitagliptin (DPP-4 inhibitor) on human platelets were shown by Gupta et al., without demonstrating a potential mechanism [248]. Cameron-Vendrig et al. investigated the role of the GLP-1 receptor on platelets in more detail. They reported that the GLP-1 analog exenatide leads to reduced formation of thrombi in mice [249]. The authors demonstrated that the GLP-1 receptor is expressed on platelets and activation of the receptor by GLP-1 analogs increases cAMP levels, which was followed by reduced thrombocyte 
aggregation in vitro and ex vivo. Sepsis causes disseminated intravasal coagulation (DIC), which is associated with thrombin generation and uncontrolled platelet activation. Under these conditions, clot formation impairs microcirculation and leads to severe end organ damage [250]. The GLP-1 analog liraglutide improved microcirculation in lung tissue by inhibition of overwhelming thrombocyte aggregation in an animal model of septic shock [247]. With our own studies, we could show that liraglutide and linagliptin as well as genetic DPP-4 deficiency prevent DIC and that this protective effect of both drugs is lost in GLP-1 receptor knockout mice (Figure 4(c)) [247]. Increased activity of cAMP/PKA signaling by GLP-1 is a potential mechanism for the antiaggregatory effects of DPP-4 inhibition and GLP-1 analogs.

3.2. SGLT2 Inhibition. Therapy with the SGLT2 inhibitor ipragliflozin not only improved hyperglycemia, hyperlipidemia, obesity, hepatic steatosis, and oxidative stress but also various parameters of inflammation in mice with T2DM or T1DM [251, 252]. The SGLT2 inhibitor dapagliflozin ameliorated glucose homeostasis and diabetic nephropathy and decreased markers of inflammation in $\mathrm{db} / \mathrm{db}$ mice [253]. Various SGLT2 inhibitors (e.g., luseogliflozin, dapagliflozin, and empagliflozin) also beneficially influenced progression of atherosclerosis by anti-inflammatory effects [254-256]. Likewise, SGLT2 inhibitors (e.g., empagliflozin, canagliflozin, ipragliflozin, and luseogliflozin) protected against NAFLD complication by decreasing inflammatory signaling [257-262], and these beneficial effects were even observed in patients with NAFLD or NASH $[263,264]$.

Empagliflozin is a potent and specific new therapeutic agent in the treatment of type 2 diabetes (T2DM) in the United States [265] and European Union (European Medicines Agency number: EMEA/H/C/002677). It is a member of a class of drugs (SGLT2i) whose mechanism of action is to inhibit the sodium-glucose cotransporter 2 (SGLT2), a protein responsible for the renal reabsorption of $>90 \%$ of the glucose from the primary urine [266]. SGLT2i are able to prevent hyperglycemic episodes and glucotoxicity by increasing the excretion of glucose $[267,268]$. Due to their insulin-independent mechanism of action, SGLT2 $i$ are not affected by deteriorating $\beta$-cell function and desensitization to insulin signaling, as is commonly seen in the elderly [269]. In recent large-scale clinical trials (EMPA-REG), empagliflozin was the only modern antidiabetic drug that was able to reduce overall cardiovascular mortality in diabetic patients at high cardiovascular risk [270, 271].

Empagliflozin is very effective in preventing glucotoxicity, including the increased methylglyoxal levels, formation of AGE, and induction of RAGE-dependent signaling that are typically seen in diabetic models. In the Zucker Diabetic Fatty (ZDF) rat model of diabetes, empagliflozin efficiently restored glycemic control. Immunohistochemical and histochemical analyses suggest that this restoration was due to the preservation of beta-cells [272]. This preservation also extended to the $\alpha$-cells of the pancreatic islets and their glucagon content, suggesting that empagliflozin may affect glucose metabolism in a dual-pronged manner (Figure 5).
Importantly, empagliflozin also improved the function of thoracic aortic segments, which coincides with linear regression analysis that revealed a significant inverse correlation between endothelial function and HbA1c. Empagliflozin treatment was also seen to have reduced oxidative stress in the aorta and blood of diabetic rats, as evident in correlations between leukocyte-dependent oxidative burst and HbAlc (Figure 5). Other's previous data showed that AGE/RAGE signaling triggers low-grade inflammation [79]. It is then possible that dynamic suppression of AGE formation and RAGE signaling, obstructing ROSinduced inflammatory pathways, and decreased epigenetic activation of inflammatory genes may all contribute to empagliflozin's anti-inflammatory properties. This is supported by our own data showing that inflammation and glucotoxicity (AGE/RAGE signaling) were epigenetically prevented by SGLT2i treatment as demonstrated by ChIP analysis. We found that there was decreased activation of NOS 2 and IFN $\gamma$ (for RAGE by trend) due to decreased $\mathrm{H} 3 \mathrm{~K} 4 \mathrm{me} 3$ in the promoter regions of the genes in kidneys of SGLT2i-treated ZDF rats, but it is not currently possible to discern whether this is a consequence of improved glucose regulation or a specific property of SGLT2i. However, additional evidence for pleiotropic activity by SGLT2i comes from improved eNOS function in hyperglycemic endothelial cells undergoing empagliflozin therapy, even independent of glucose lowering. This effect was also shared by DPP-4 inhibitor sitagliptin and partially by the RAGE antagonist FPS-ZM1 [272].

We reported similar antidiabetic, antioxidant, antiinflammatory, and vasculoprotective effects of empagliflozin treatment in rats with streptozotocin- (STZ-) induced T1DM [267]. Treatment with empagliflozin importantly reversed the proinflammatory phenotype of diabetes as well as the glucotoxicity (AGE/RAGE). In diabetic rats, empagliflozin expectedly reduced blood glucose levels but it also normalized endothelial function in aortic rings and reduced oxidative stress in aortic vessels (dihydroethidium staining) and in blood (phorbol ester/zymosan A-stimulated chemiluminescence).

\section{Conclusion}

Cardiovascular disease is the leading cause of mortality in industrialized nations. Smoking, unhealthy nutrition, aging population, lack of physical activity, arterial hypertension, or diabetes promote cardiovascular disease like myocardial infarction or stroke (Figure 6). It is multifactorial and encompasses a multitude of mechanisms, such as eNOS uncoupling, reactive oxygen species formation, abnormal calcium homeostasis, signaling associated with a deleterious phosphorylation pattern, and futile counterregulatory mechanisms at the humoral, cellular, and tissue levels. Previous data also implicate immune cells and inflammatory signaling as essential contributors to cardiovascular disease (for review, see [7]). Protective effects are discussed for important endogenous antioxidant enzymes as well as for modern drugs with pleiotropic antioxidant and antiinflammatory properties. Three highly promising classes 


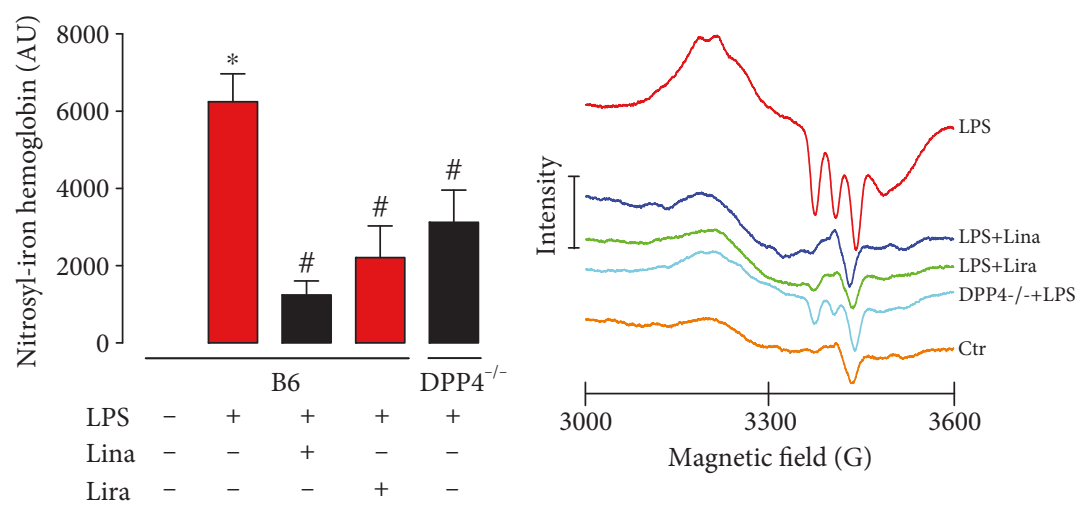

(a)

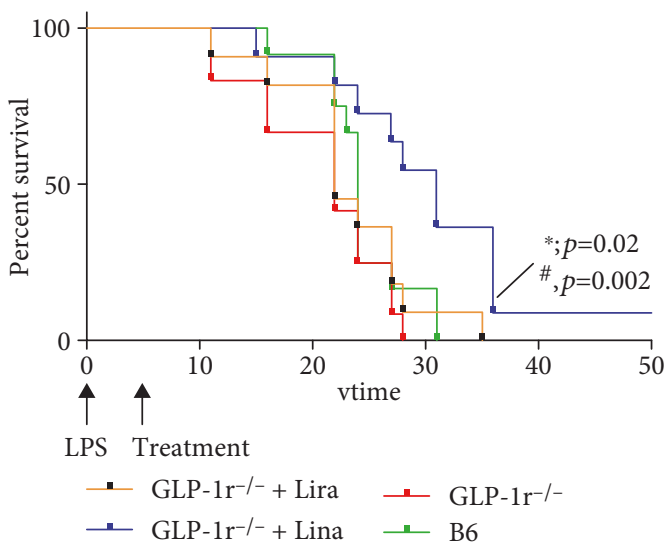

(b)

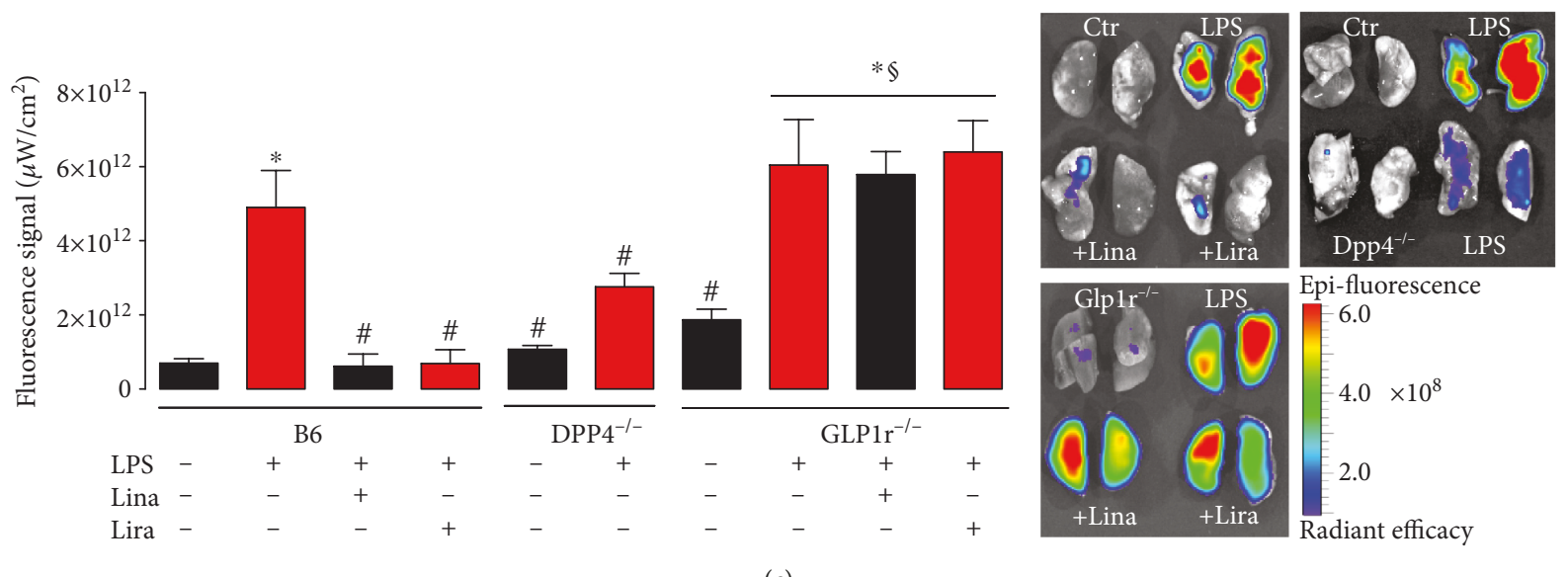

(c)

FIGURE 4: Antioxidant, anti-inflammatory, and vasculoprotective effects by the DPP-4 inhibitor or GLP-1 analog therapy in septic mice. (a) Whole blood $\mathrm{Hb}$-NO levels were determined by electron paramagnetic resonance spectroscopy as a read-out of iNOS activity. (b) Mortality of endotoxemic mice was assessed by Kaplan-Meier curves recording the survival in dependence of time. $17.5 \mathrm{mg} / \mathrm{kg}$ LPS or solvent was administrated by i.p. injection. DPP-4 inhibitor (Lina: $5 \mathrm{mg} / \mathrm{kg} / \mathrm{d}$ s.c. for $3 \mathrm{~d}$ ) and GLP-1 analogue (Lira: $200 \mu \mathrm{g} / \mathrm{kg} / \mathrm{d}$ s.c. for $3 \mathrm{~d}$ ) treatment was started $6 \mathrm{~h}$ after the induction of endotoxemia. (c) Microvascular thrombosis was detected by fluorescence imaging using fluorescent microbeads in endotoxemic wild-type mice, DPP- $4^{-/-}$mice, and GLP1 $\mathrm{r}^{-/}$mice. Representative images of lungs are shown beside the quantification. The data are mean \pm SEM from 6-18 (a), 12 (b), or 4-6 (c) mice/group. ${ }^{*} p<0.05$ vs. B6; ${ }^{\#} p<0.05$ vs. B6+LPS; ${ }^{\$} p<0.05$ vs. GLP-1 $\mathrm{r}^{-/-}$. Adopted from Steven et al. [247] (with permission from John Wiley and Sons. Copyright @ 2016, John Wiley and Sons).

of modern antidiabetic cardiovascular drugs are highlighted (SGLT2 inhibitors, DPP-4 inhibitors, and GLP-1 analogs). The present review defines the current knowledge connecting the immune system with cardiovascular disease, allowing not only for more integrative ther- apies using multidisciplinary expertise but also potential treatments for the associated comorbidities. In the future, the modulation of epigenetic pathways may represent an important future strategy due to the observed global changes in $\mathrm{H} 3 \mathrm{~K} 4$ trimethylation which are associated with 


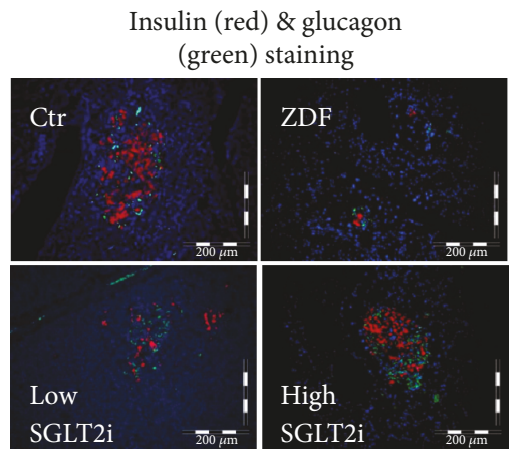

(a)

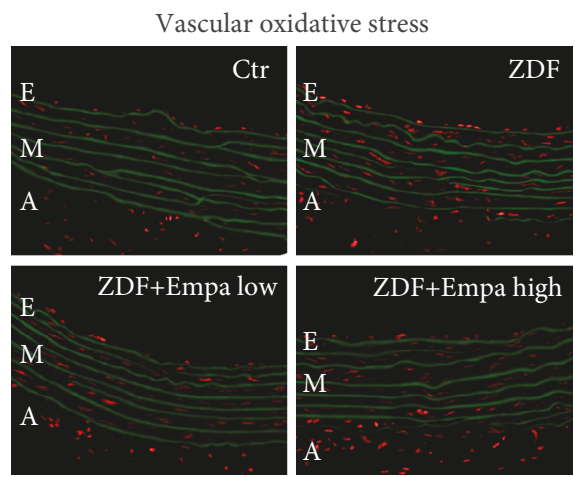

(c)

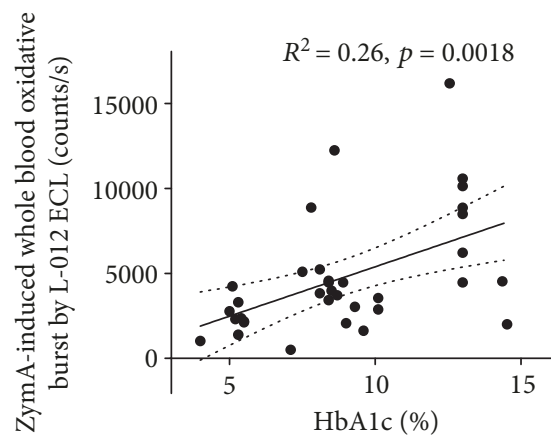

(e)

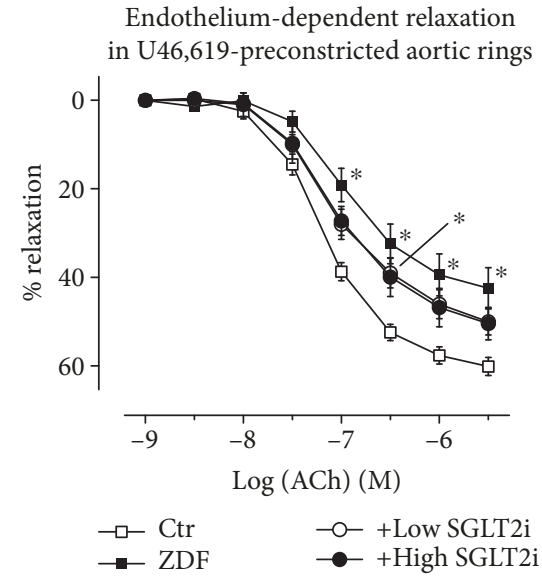

(b)

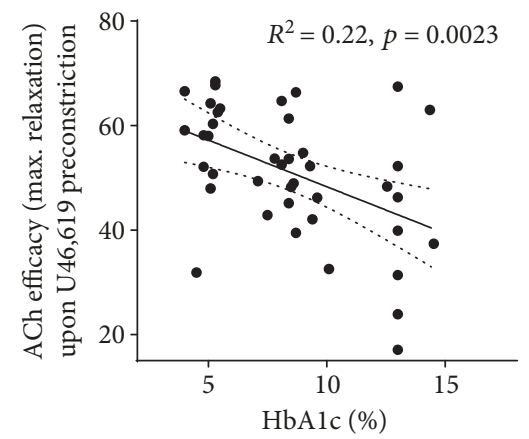

(d)

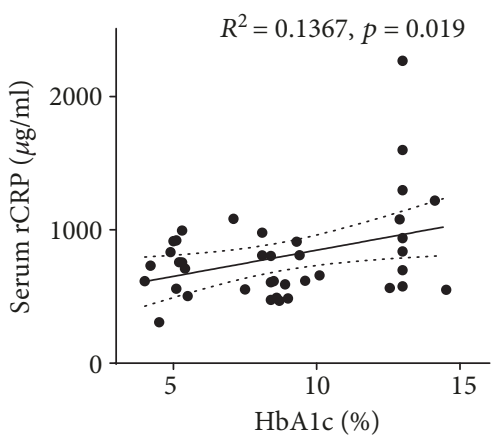

(f)

FIgURE 5: Antioxidant, anti-inflammatory, and vasculoprotective effects by empagliflozin therapy in ZDF rats. (a) Representative (immuno)histochemical stainings of pancreatic tissue for insulin, glucagon, and nuclei using fluorescent antibodies and dyes. (b) Improvement of endothelium-dependent relaxation by the vasodilator acetylcholine (ACh) in U46,619-preconstricted aortic ring segments. (c) Dihydroethidium (DHE, $1 \mu \mathrm{M}$ ) fluorescence microtopography was used to assess the effects of SGLT2i treatment on whole vascular wall ROS production, and representative microscope images are shown (red fluorescence indicates ROS formation whereas green fluorescence represents basal laminae autofluorescence). Linear regression analysis for correlations between HbAlc and endothelial function (ACh efficacy, d), zymosan A-induced whole blood oxidative burst (e), and serum CRP levels (f) using a total of $35-41$ rats. ${ }^{*} p<0.05$ vs. the control. Adopted from Steven et al. [272] (with permission from Elsevier. (c) 2017 The Authors. Published by Elsevier B.V.).

high body weight and T2DM [273]. Additionally, increased promoter $\mathrm{H} 3 \mathrm{~K} 9 \mathrm{me} 2$ in genes associated with autoimmune and inflammation-related pathways (transforming growth factor-beta $(\mathrm{TGF} \beta)$, nuclear factor- $\kappa \mathrm{B}$, p38 mitogen-activated protein kinase, toll-like receptor, and interleukin-6) are present in diabetic patients implying anti-inflammatory potential of epigenetic modulators
[274]. An additional field open for investigation lies in the comparison of drugs with similar primary pharmacological effects (e.g., blood pressure lowering), with and without known pleiotropic effects (e.g., anti-inflammatory or antioxidant components) in order to discern the role of this pleiotropy. This data would be valuable in treating patients, especially in conjunction with prescreening for 


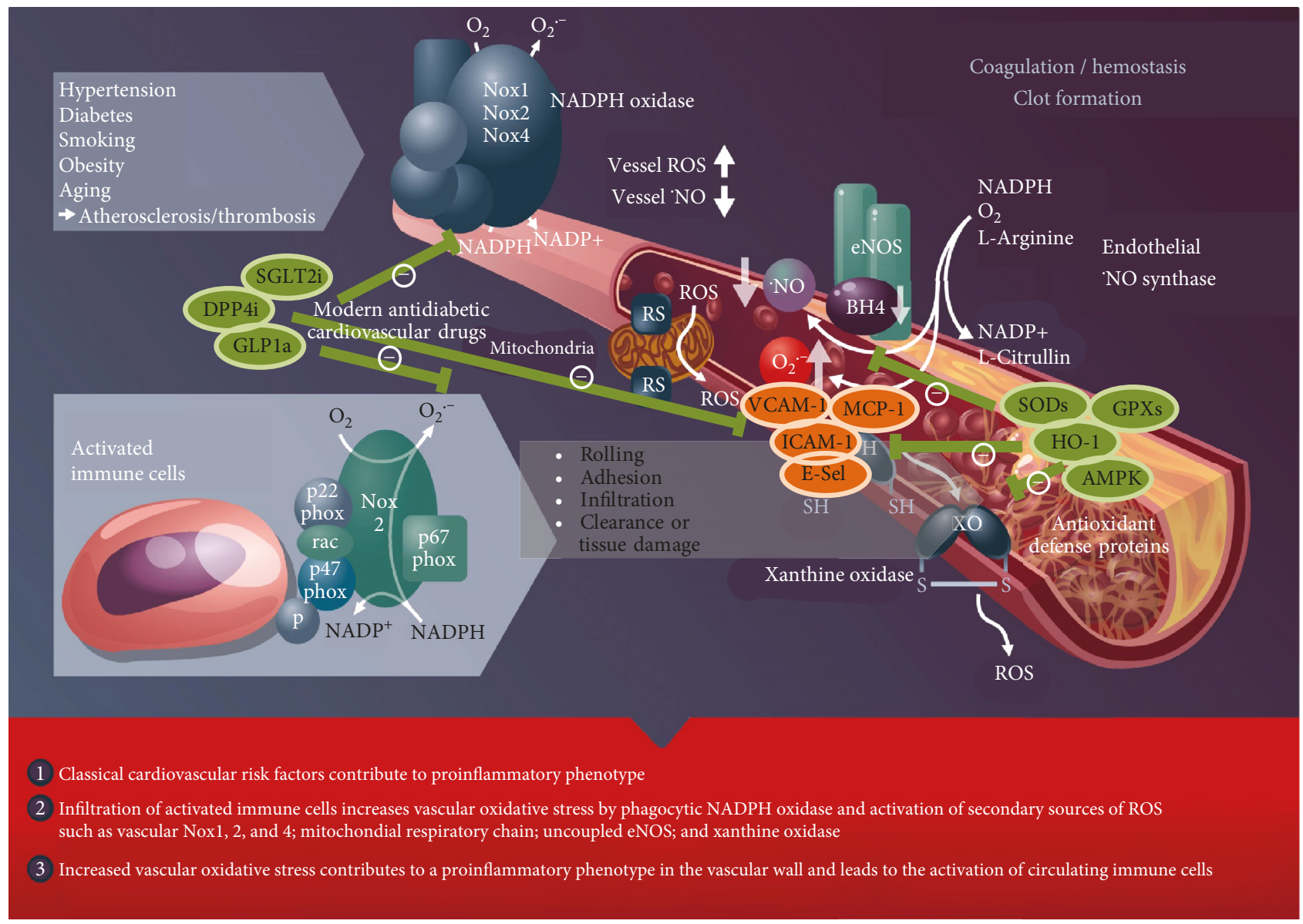

FIGURE 6: Oxidative stress and inflammation trigger atherothrombosis. The scheme illustrates the activation of immune cells and recruitment to vascular tissues by classical cardiovascular risk factors, leading to the activation of secondary vascular ROS sources such as NADPH oxidase (Nox1, Nox2, and Nox4), xanthine oxidase (conversion of the dehydrogenase (XDH) to the oxidase (XO) form), mitochondria (via mitochondrial redox switches (RS)), and uncoupled eNOS (oxidative depletion of tetrahydrobiopterin (BH4) and other redox switches), all of which contribute to vascular dysfunction, progression of atherosclerosis, and thrombus formation. Adhesion molecules and chemokines (VCAM-1, ICAM-1, E-selectin, and MCP-1) play an important role for leukocyte recruitment to vascular tissues leading to the secondary damage. Endogenous antioxidant defense proteins (e.g., superoxide dismutases, glutathione peroxidases, heme oxygenase-1, and AMP-activated protein kinase) interfere with oxidative damage and redox-dependent inflammatory processes. Also, modern antidiabetic cardiovascular drugs (e.g., SGLT2 inhibitors, DPP-4 inhibitors, and GLP-1 analogs) suppress inflammatory and adverse redox pathways and thereby decrease cardiovascular risk. Modified from Steven et al. [8]. Open access article distributed under the Creative Commons Attribution License (CC BY 4.0).

markers of inflammation and/or oxidative stress-paving the way toward personalized medicine in cardiovascular disease therapy.

\section{Conflicts of Interest}

T.M. and A.D. received research grants from Boehringer Ingelheim Pharma $\mathrm{GmbH} \& \mathrm{Co}$. KG. The other authors declare that they have no competing interests in connection with this manuscript.

\section{Authors' Contributions}

S. Steven, K. Frenis, T. Münzel, and A. Daiber drafted the manuscript, and M. Oelze, S. Kalinovic, M. Kuntic, M.T. Bayo Jimenez, K. Vujacic-Mirski, J. Helmstädter, and S. Kröller-Schön made critical revisions.

\section{Acknowledgments}

S.S., T.M., and A.D. were supported by a vascular biology research grant from the Boehringer Ingelheim Stiftung for the collaborative research group "Novel and Neglected Cardiovascular Risk Factors: Molecular Mechanisms and Therapeutic Implications." K.F., S.K., M.K., M.T.B.J., and K.V.-M. hold PhD stipends of the TransMed PhD Program at the University Medical Center Mainz, and their positions are funded by a vascular biology research grant from the Boehringer Ingelheim Stiftung for the collaborative research group "Novel and Neglected Cardiovascular Risk Factors: Molecular Mechanisms and Therapeutic Implications." In addition, we are grateful for the continuous support by the Foundation Heart of Mainz, Mainz, Germany. T.M. is PI of the DZHK (German Center for Cardiovascular Research), Partner Site Rhine-Main, Mainz, Germany. The original animal studies were supported by 
vascular biology research grants from Boehringer Ingelheim Pharma GmbH \& Co. KG, Ingelheim, Germany (A.D. and T.M.).

\section{References}

[1] L. Becker, K. Prado, M. Foppa et al., "Endothelial dysfunction assessed by brachial artery ultrasound in severe sepsis and septic shock," Journal of Critical Care, vol. 27, no. 3, pp. 316.e9-316.e14, 2012.

[2] R. Bergholm, M. Leirisalo-Repo, S. Vehkavaara, S. Makimattila, M. R. Taskinen, and H. Yki-Jarvinen, "Impaired responsiveness to NO in newly diagnosed patients with rheumatoid arthritis," Arteriosclerosis, Thrombosis, and Vascular Biology, vol. 22, no. 10, pp. 1637-1641, 2002.

[3] S. Hansel, G. Lassig, F. Pistrosch, and J. Passauer, "Endothelial dysfunction in young patients with long-term rheumatoid arthritis and low disease activity," Atherosclerosis, vol. 170, no. 1, pp. 177-180, 2003.

[4] N. N. Mehta, R. S. Azfar, D. B. Shin, A. L. Neimann, A. B. Troxel, and J. M. Gelfand, "Patients with severe psoriasis are at increased risk of cardiovascular mortality: cohort study using the General Practice Research Database," European Heart Journal, vol. 31, no. 8, pp. 1000-1006, 2010.

[5] R. M. A. Henry, I. Ferreira, P. J. Kostense et al., "Type 2 diabetes is associated with impaired endothelium-dependent, flow-mediated dilation, but impaired glucose metabolism is not; the Hoorn Study," Atherosclerosis, vol. 174, no. 1, pp. 49-56, 2004.

[6] P. M. Ridker, C. P. Cannon, D. Morrow et al., "C-reactive protein levels and outcomes after statin therapy," The New England Journal of Medicine, vol. 352, no. 1, pp. 20-28, 2005.

[7] S. Karbach, P. Wenzel, A. Waisman, T. Munzel, and A. Daiber, "eNOS uncoupling in cardiovascular diseases the role of oxidative stress and inflammation," Current Pharmaceutical Design, vol. 20, no. 22, pp. 3579-3594, 2014.

[8] S. Steven, T. Munzel, and A. Daiber, "Exploiting the pleiotropic antioxidant effects of established drugs in cardiovascular disease," International Journal of Molecular Sciences, vol. 16, no. 8, pp. 18185-18223, 2015.

[9] A. Daiber, S. Steven, A. Weber et al., "Targeting vascular (endothelial) dysfunction," British Journal of Pharmacology, vol. 174, no. 12, pp. 1591-1619, 2017.

[10] K. N. Prasad, "Simultaneous activation of Nrf2 and elevation of antioxidant compounds for reducing oxidative stress and chronic inflammation in human Alzheimer's disease," Mechanisms of Ageing and Development, vol. 153, pp. 41-47, 2016.

[11] P. D. Wes, F. A. Sayed, F. Bard, and L. Gan, "Targeting microglia for the treatment of Alzheimer's disease," Glia, vol. 64, no. 10, pp. 1710-1732, 2016.

[12] A. Katsumoto, H. Takeuchi, K. Takahashi, and F. Tanaka, "Microglia in Alzheimer's disease: risk factors and inflammation," Frontiers in Neurology, vol. 9, p. 978, 2018.

[13] O. Gorelenkova Miller, J. B. Behring, S. L. Siedlak et al., "Upregulation of glutaredoxin-1 activates microglia and promotes neurodegeneration: implications for Parkinson's disease," Antioxidants \& Redox Signaling, vol. 25, no. 18, pp. 967-982, 2016.

[14] P. Troncoso-Escudero, A. Parra, M. Nassif, and R. L. Vidal, "Outside in: unraveling the role of neuroinflammation in the progression of Parkinson's disease," Frontiers in Neurology, vol. 9, p. 860, 2018.

[15] S. S. Lim, T. Vos, A. D. Flaxman et al., "A comparative risk assessment of burden of disease and injury attributable to 67 risk factors and risk factor clusters in 21 regions, 1990-2010: a systematic analysis for the Global Burden of Disease Study 2010," The Lancet, vol. 380, no. 9859, pp. 2224-2260, 2012.

[16] C. J. Murray, M. Ezzati, A. D. Flaxman et al., "GBD 2010: design, definitions, and metrics," The Lancet, vol. 380, no. 9859 , pp. 2063-2066, 2012.

[17] A. J. Cohen, M. Brauer, R. Burnett et al., "Estimates and 25year trends of the global burden of disease attributable to ambient air pollution: an analysis of data from the Global Burden of Diseases Study 2015," The Lancet, vol. 389, no. 10082, pp. 1907-1918, 2017.

[18] R. A. Iseme, M. McEvoy, B. Kelly et al., "A role for autoantibodies in atherogenesis," Cardiovascular Research, vol. 113, no. 10, pp. 1102-1112, 2017.

[19] S. Kaptoge, S. R. K. Seshasai, P. Gao et al., "Inflammatory cytokines and risk of coronary heart disease: new prospective study and updated meta-analysis," European Heart Journal, vol. 35, no. 9, pp. 578-589, 2014.

[20] G. K. Hansson, "Inflammation, atherosclerosis, and coronary artery disease," The New England Journal of Medicine, vol. 352, no. 16, pp. 1685-1695, 2005.

[21] D. T. Kelly, "Paul Dudley White international lecture. Our future society. A global challenge," Circulation, vol. 95, no. 11, pp. 2459-2464, 1997.

[22] M. Cesari, G. Onder, A. Russo et al., "Comorbidity and physical function: results from the aging and longevity study in the Sirente geographic area (ilSIRENTE study)," Gerontology, vol. 52, no. 1, pp. 24-32, 2006.

[23] R. Yancik, W. Ershler, W. Satariano, W. Hazzard, H. J. Cohen, and L. Ferrucci, "Report of the national institute on aging task force on comorbidity," The Journals of Gerontology Series A: Biological Sciences and Medical Sciences, vol. 62, no. 3, pp. 275-280, 2007.

[24] G. D. Wieland, "From bedside to bench: research in comorbidity and aging," Science of Aging Knowledge Environment, vol. 2005, no. 39, p. pe29, 2005.

[25] E. G. Lakatta and D. Levy, “Arterial and cardiac aging: major shareholders in cardiovascular disease enterprises: part I: aging arteries: a "set up" for vascular disease," Circulation, vol. 107, no. 1, pp. 139-146, 2003.

[26] M. D. Herrera, C. Mingorance, R. Rodriguez-Rodriguez, and M. Alvarez de Sotomayor, "Endothelial dysfunction and aging: an update," Ageing Research Reviews, vol. 9, no. 2, pp. 142-152, 2010.

[27] D. R. Seals, K. L. Jablonski, and A. J. Donato, "Aging and vascular endothelial function in humans," Clinical Science, vol. 120, no. 9, pp. 357-375, 2011.

[28] H. Tanaka, F. A. Dinenno, and D. R. Seals, "Age-related increase in femoral intima-media thickness in healthy humans," Arteriosclerosis, Thrombosis, and Vascular Biology, vol. 20, no. 9, p. 2172, 2000.

[29] B. Bischoff, S. Silber, B. M. Richartz et al., "Inadequate medical treatment of patients with coronary artery disease by primary care physicians in Germany," Clinical Research in Cardiology, vol. 95, no. 8, pp. 405-412, 2006.

[30] R. T. Ras, M. T. Streppel, R. Draijer, and P. L. Zock, "Flow-mediated dilation and cardiovascular risk 
prediction: a systematic review with meta-analysis," International Journal of Cardiology, vol. 168, no. 1, pp. 344351, 2013.

[31] M. Shechter, A. Shechter, H. Hod et al., "Brachial artery endothelial function predicts platelet function in control subjects and in patients with acute myocardial infarction," Platelets, vol. 23, no. 3, pp. 202-210, 2012.

[32] K. B. Vallbracht, P. L. Schwimmbeck, B. Seeberg, U. Kuhl, and H. P. Schultheiss, "Endothelial dysfunction of peripheral arteries in patients with immunohistologically confirmed myocardial inflammation correlates with endothelial expression of human leukocyte antigens and adhesion molecules in myocardial biopsies," Journal of the American College of Cardiology, vol. 40, no. 3, pp. 515-520, 2002.

[33] M. Krikke, K. Tesselaar, J. E. Arends et al., "Maraviroc intensification improves endothelial function in abacavir-treated patients, an open-label randomized cross-over pilot study," Infectious Diseases and Therapy, vol. 5, no. 3, pp. 389-404, 2016.

[34] L. Lind, M. Sarabi, J. Millgard, T. Kahan, and M. Edner, "Endothelium-dependent vasodilation and structural and functional changes in the cardiovascular system are dependent on age in healthy subjects," Clinical Physiology, vol. 19, no. 5, pp. 400-409, 1999.

[35] M. El Assar, J. Angulo, and L. Rodriguez-Manas, "Oxidative stress and vascular inflammation in aging," Free Radical Biology \& Medicine, vol. 65, pp. 380-401, 2013.

[36] E. Crimi, L. J. Ignarro, and C. Napoli, "Microcirculation and oxidative stress," Free Radical Research, vol. 41, no. 12, pp. 1364-1375, 2007.

[37] A. L. Burnett, "The role of nitric oxide in erectile dysfunction: implications for medical therapy," The Journal of Clinical Hypertension, vol. 8, no. 12, Supplement 4, pp. 53-62, 2006.

[38] A. Csiszar, J. Toth, J. Peti-Peterdi, and Z. Ungvari, “The aging kidney: role of endothelial oxidative stress and inflammation," Acta Physiologica Hungarica, vol. 94, no. 1-2, pp. 107-115, 2007.

[39] J. M. Price, A. Hellermann, G. Hellermann, and E. T. Sutton, "Aging enhances vascular dysfunction induced by the Alzheimer's peptide $\beta$-amyloid," Neurological Research, vol. 26, no. 3, pp. 305-311, 2004.

[40] H. R. Coleman, C. C. Chan, F. L. Ferris III, and E. Y. Chew, "Age-related macular degeneration," The Lancet, vol. 372, no. 9652, pp. 1835-1845, 2008.

[41] W. G. Mayhan, D. M. Arrick, G. M. Sharpe, and H. Sun, "Age-related alterations in reactivity of cerebral arterioles: role of oxidative stress," Microcirculation, vol. 15, no. 3, pp. 225-236, 2008.

[42] J. Militante and J. B. Lombardini, “Age-related retinal degeneration in animal models of aging: possible involvement of taurine deficiency and oxidative stress," Neurochemical Research, vol. 29, no. 1, pp. 151-160, 2004.

[43] R. Fischer and O. Maier, "Interrelation of oxidative stress and inflammation in neurodegenerative disease: role of TNF," Oxidative Medicine and Cellular Longevity, vol. 2015, Article ID 610813, 18 pages, 2015.

[44] J. Blasiak, G. Petrovski, Z. Vereb, A. Facsko, and K. Kaarniranta, "Oxidative stress, hypoxia, and autophagy in the neovascular processes of age-related macular degeneration," BioMed Research International, vol. 2014, Article ID 768026, 7 pages, 2014.
[45] A. Daiber, J. Kienhoefer, R. Zee et al., "The role of mitochondrial reactive oxygen species formation for age-induced vascular dysfunction," in Aging and Age-Related Disorders, S. Bondy and K. Maiese, Eds., pp. 237-257, Humana Press, 2010.

[46] Y. Mikhed, A. Daiber, and S. Steven, "Mitochondrial oxidative stress, mitochondrial DNA damage and their role in age-related vascular dysfunction," International Journal of Molecular Sciences, vol. 16, no. 7, pp. 15918-15953, 2015.

[47] M. Gerhard, M. A. Roddy, S. J. Creager, and M. A. Creager, "Aging progressively impairs endothelium-dependent vasodilation in forearm resistance vessels of humans," Hypertension, vol. 27, no. 4, pp. 849-853, 1996.

[48] P. Jousilahti, E. Vartiainen, J. Tuomilehto, and P. Puska, "Sex, age, cardiovascular risk factors, and coronary heart disease: a prospective follow-up study of 14786 middle-aged men and women in Finland," Circulation, vol. 99, no. 9, pp. 11651172, 1999.

[49] E. A. Miles, D. Rees, T. Banerjee et al., "Age-related increases in circulating inflammatory markers in men are independent of BMI, blood pressure and blood lipid concentrations," Atherosclerosis, vol. 196, no. 1, pp. 298-305, 2008.

[50] L. Ferrucci, A. Corsi, F. Lauretani et al., "The origins of agerelated proinflammatory state," Blood, vol. 105, no. 6, pp. 2294-2299, 2005.

[51] P. Soysal, B. Stubbs, P. Lucato et al., "Inflammation and frailty in the elderly: a systematic review and meta-analysis," Ageing Research Reviews, vol. 31, pp. 1-8, 2016.

[52] "WHO report Diabetes 2016," in https://apps.who.int/iris/ bitstream/handle/10665/204871/9789241565257_eng .pdf? sequence $=1$.

[53] E. Standl and O. Schnell, "A new look at the heart in diabetes mellitus: from ailing to failing," Diabetologia, vol. 43, no. 12, pp. 1455-1469, 2000.

[54] C. B. Granger, R. M. Califf, S. Young et al., "Outcome of patients with diabetes mellitus and acute myocardial infarction treated with thrombolytic agents," Journal of the American College of Cardiology, vol. 21, no. 4, pp. 920-925, 1993.

[55] W. Otter, S. Kleybrink, W. Doering, E. Standl, and O. Schnell, "Hospital outcome of acute myocardial infarction in patients with and without diabetes mellitus," Diabetic Medicine, vol. 21, no. 2, pp. 183-187, 2004.

[56] B. Manns, B. Hemmelgarn, M. Tonelli et al., "Population based screening for chronic kidney disease: cost effectiveness study," BMJ, vol. 341, article c5869, 2010.

[57] J. A. Vita, C. B. Treasure, E. G. Nabel et al., "Coronary vasomotor response to acetylcholine relates to risk factors for coronary artery disease," Circulation, vol. 81, no. 2, pp. 491-497, 1990.

[58] A. Calver, J. Collier, and P. Vallance, "Inhibition and stimulation of nitric oxide synthesis in the human forearm arterial bed of patients with insulin-dependent diabetes," The Journal of Clinical Investigation, vol. 90, no. 6, pp. 2548-2554, 1992.

[59] J. A. Panza, A. A. Quyyumi, J. E. Brush Jr., and S. E. Epstein, "Abnormal endothelium-dependent vascular relaxation in patients with essential hypertension," The New England Journal of Medicine, vol. 323, no. 1, pp. 22-27, 1990.

[60] T. Nakagami, Q. Qiao, J. Tuomilehto et al., "Screen-detected diabetes, hypertension and hypercholesterolemia as predictors of cardiovascular mortality in five populations of Asian origin: the DECODA study," European Journal of 
Cardiovascular Prevention \& Rehabilitation, vol. 13, no. 4, pp. 555-561, 2006.

[61] J. de Jager, J. M. Dekker, A. Kooy et al., "Endothelial dysfunction and low-grade inflammation explain much of the excess cardiovascular mortality in individuals with type 2 diabetes: the Hoorn Study," Arteriosclerosis, Thrombosis, and Vascular Biology, vol. 26, no. 5, pp. 1086-1093, 2006.

[62] Y. Wang, J. Wang, Z. Deng et al., "Chronic osteomyelitis increases the incidence of type 2 diabetes in humans and mice," International Journal of Biological Sciences, vol. 13, no. 9, pp. 1192-1202, 2017.

[63] X. Wang, W. Bao, J. Liu et al., "Inflammatory markers and risk of type 2 diabetes: a systematic review and meta-analysis," Diabetes Care, vol. 36, no. 1, pp. 166-175, 2013.

[64] H. C. Gerstein, K. Swedberg, J. Carlsson et al., "The hemoglobin A1c level as a progressive risk factor for cardiovascular death, hospitalization for heart failure, or death in patients with chronic heart failure: an analysis of the Candesartan in Heart failure: Assessment of Reduction in Mortality and Morbidity (CHARM) program," Archives of Internal Medicine, vol. 168, no. 15, pp. 1699-1704, 2008.

[65] J. F. Keaney Jr. and J. Loscalzo, "Diabetes, oxidative stress, and platelet activation," Circulation, vol. 99, no. 2, pp. 189191, 1999.

[66] B. Lipinski, "Pathophysiology of oxidative stress in diabetes mellitus," Journal of Diabetes and its Complications, vol. 15, no. 4, pp. 203-210, 2001.

[67] K. K. Griendling and G. A. FitzGerald, "Oxidative stress and cardiovascular injury: part II: animal and human studies," Circulation, vol. 108, no. 17, pp. 2034-2040, 2003.

[68] T. Heitzer, T. Schlinzig, K. Krohn, T. Meinertz, and T. Munzel, "Endothelial dysfunction, oxidative stress, and risk of cardiovascular events in patients with coronary artery disease," Circulation, vol. 104, no. 22, pp. 2673-2678, 2001.

[69] G. D. Johnston, "Ulster says 'NO'; explosion, resistance and tolerance. Nitric oxide and the actions of organic nitrates," The Ulster Medical Journal, vol. 67, no. 2, pp. 79-90, 1998.

[70] J. Uitto, A. J. Perejda, G. A. Grant, E. A. Rowold, C. Kilo, and J. R. Williamson, "Glycosylation of human glomerular basement membrane collagen: increased content of hexose in ketoamine linkage and unaltered hydroxylysine-Oglycosides in patients with diabetes," Connective Tissue Research, vol. 10, no. 3-4, pp. 287-296, 1982.

[71] R. Bucala, K. J. Tracey, and A. Cerami, "Advanced glycosylation products quench nitric oxide and mediate defective endothelium-dependent vasodilatation in experimental diabetes," Journal of Clinical Investigation, vol. 87, no. 2, pp. 432-438, 1991.

[72] M. Sun, M. Yokoyama, T. Ishiwata, and G. Asano, "Deposition of advanced glycation end products (AGE) and expression of the receptor for AGE in cardiovascular tissue of the diabetic rat," International Journal of Experimental Pathology, vol. 79, no. 4, pp. 207-222, 1998.

[73] J. L. Wautier, M. P. Wautier, A. M. Schmidt et al., "Advanced glycation end products (AGEs) on the surface of diabetic erythrocytes bind to the vessel wall via a specific receptor inducing oxidant stress in the vasculature: a link between surface-associated AGEs and diabetic complications," Proceedings of the National Academy of Sciences of the United States of America, vol. 91, no. 16, pp. 7742-7746, 1994.
[74] M. P. Wautier, O. Chappey, S. Corda, D. M. Stern, A. M. Schmidt, and J. L. Wautier, "Activation of NADPH oxidase by AGE links oxidant stress to altered gene expression via RAGE," American Journal of Physiology-Endocrinology and Metabolism, vol. 280, no. 5, pp. E685-E694, 2001.

[75] M. T. Coughlan, D. R. Thorburn, S. A. Penfold et al., "RAGEinduced cytosolic ROS promote mitochondrial superoxide generation in diabetes," Journal of the American Society of Nephrology, vol. 20, no. 4, pp. 742-752, 2009.

[76] T. Nishikawa, D. Edelstein, X. L. Du et al., "Normalizing mitochondrial superoxide production blocks three pathways of hyperglycaemic damage," Nature, vol. 404, no. 6779, pp. 787-790, 2000.

[77] E. Schulz, P. Wenzel, T. Munzel, and A. Daiber, "Mitochondrial redox signaling: interaction of mitochondrial reactive oxygen species with other sources of oxidative stress," Antioxidants \& Redox Signaling, vol. 20, no. 2, pp. 308-324, 2014.

[78] S. Kroller-Schon, S. Steven, S. Kossmann et al., "Molecular mechanisms of the crosstalk between mitochondria and NADPH oxidase through reactive oxygen species-studies in white blood cells and in animal models," Antioxidants \& Redox Signaling, vol. 20, no. 2, pp. 247-266, 2014.

[79] L. G. Bucciarelli, T. Wendt, W. Qu et al., "RAGE blockade stabilizes established atherosclerosis in diabetic apolipoprotein E-null mice," Circulation, vol. 106, no. 22, pp. 28272835, 2002.

[80] S. Kumar, S. Prasad, and S. L. Sitasawad, "Multiple antioxidants improve cardiac complications and inhibit cardiac cell death in streptozotocin-induced diabetic rats," PLoS One, vol. 8, no. 7, article e67009, 2013.

[81] P. D. Taylor and L. Poston, "The effect of hyperglycaemia on function of rat isolated mesenteric resistance artery," British Journal of Pharmacology, vol. 113, no. 3, pp. 801-808, 1994.

[82] M. Oelze, S. Schuhmacher, and A. Daiber, "Organic nitrates and nitrate resistance in diabetes: the role of vascular dysfunction and oxidative stress with emphasis on antioxidant properties of pentaerithrityl tetranitrate," Experimental Diabetes Research, vol. 2010, Article ID 213176, 13 pages, 2010.

[83] M. Brownlee, "Biochemistry and molecular cell biology of diabetic complications," Nature, vol. 414 , no. 6865 , pp. 813820, 2001.

[84] A. H. Barnett, S. C. Bain, P. Bouter et al., “Angiotensin-receptor blockade versus converting-enzyme inhibition in type 2 diabetes and nephropathy," The New England Journal of Medicine, vol. 351, no. 19, pp. 1952-1961, 2004.

[85] M. J. Crespo and D. C. Dunbar, "Enalapril improves vascular and cardiac function in streptozotocin-diabetic rats," Cellular and Molecular Biology, vol. 49, no. 8, pp. 13111318, 2003.

[86] A. Ceriello, R. Assaloni, R. Da Ros et al., "Effect of atorvastatin and irbesartan, alone and in combination, on postprandial endothelial dysfunction, oxidative stress, and inflammation in type 2 diabetic patients," Circulation, vol. 111, no. 19, pp. 2518-2524, 2005.

[87] H. Ishii, D. Koya, and G. L. King, "Protein kinase C activation and its role in the development of vascular complications in diabetes mellitus," Journal of Molecular Medicine, vol. 76, no. 1, pp. 21-31, 1998.

[88] D. Koya and G. L. King, "Protein kinase C activation and the development of diabetic complications," Diabetes, vol. 47, no. 6, pp. 859-866, 1998. 
[89] T. Inoguchi, P. Li, F. Umeda et al., "High glucose level and free fatty acid stimulate reactive oxygen species production through protein kinase C-dependent activation of $\mathrm{NAD}(\mathrm{P}) \mathrm{H}$ oxidase in cultured vascular cells," Diabetes, vol. 49, no. 11, pp. 1939-1945, 2000.

[90] D. G. Harrison, Hua Cai, U. Landmesser, and K. K. Griendling, "The pickering lecture British Hypertension Society, 10th September 2002: interactions of angiotensin II with $\mathrm{NAD}(\mathrm{P}) \mathrm{H}$ oxidase, oxidant stress and cardiovascular disease," Journal of the Renin-Angiotensin-Aldosterone System, vol. 4, no. 2, pp. 51-61, 2003.

[91] J. H. Oak and H. Cai, "Attenuation of angiotensin II signaling recouples eNOS and inhibits nonendothelial NOX activity in diabetic mice,” Diabetes, vol. 56, no. 1, pp. 118-126, 2007.

[92] R. Nosadini and G. Tonolo, "The role of the renin angiotensin hormonal system in the metabolic syndrome and type 2 diabetes," Nutrition, Metabolism and Cardiovascular Diseases, vol. 14, no. 2, pp. 88-93, 2004.

[93] G. C. Fonarow, "An approach to heart failure and diabetes mellitus," The American Journal of Cardiology, vol. 96, no. 4, pp. 47-52, 2005.

[94] M. Lorenzi and E. Cagliero, "Pathobiology of endothelial and other vascular cells in diabetes mellitus: Call for data," Diabetes, vol. 40, no. 6, pp. 653-659, 1991.

[95] Y. Ohara, T. E. Peterson, and D. G. Harrison, "Hypercholesterolemia increases endothelial superoxide anion production," The Journal of Clinical Investigation, vol. 91, no. 6, pp. 2546-2551, 1993.

[96] D. G. Harrison and Y. Ohara, "Physiologic consequences of increased vascular oxidant stresses in hypercholesterolemia and atherosclerosis: implications for impaired vasomotion," The American Journal of Cardiology, vol. 75, no. 6, pp. 75B81B, 1995.

[97] K. K. Griendling and G. A. FitzGerald, "Oxidative stress and cardiovascular injury: part I: basic mechanisms and in vivo monitoring of ROS," Circulation, vol. 108, no. 16, pp. 19121916, 2003.

[98] H. Sies, "Oxidative stress: a concept in redox biology and medicine,” Redox Biology, vol. 4, pp. 180-183, 2015.

[99] A. Daiber, M. Oelze, S. Daub et al., "Vascular redox signaling, redox switches in endothelial nitric oxide synthase (eNOS uncoupling), and endothelial dysfunction," in Systems Biology of Free Radicals and Antioxidants, I. Laher, Ed., pp. 1177-1211, Springer, Berlin, Heidelberg, 2014.

[100] P. Wenzel, S. Kossmann, T. Munzel, and A. Daiber, "Redox regulation of cardiovascular inflammation - immunomodulatory function of mitochondrial and Nox-derived reactive oxygen and nitrogen species," Free Radical Biology \& Medicine, vol. 109, pp. 48-60, 2017.

[101] A. Daiber, F. Di Lisa, M. Oelze et al., "Crosstalk of mitochondria with NADPH oxidase via reactive oxygen and nitrogen species signalling and its role for vascular function," British Journal of Pharmacology, vol. 174, no. 12, pp. 1670-1689, 2017.

[102] A. Daiber, "Redox signaling (cross-talk) from and to mitochondria involves mitochondrial pores and reactive oxygen species," Biochimica et Biophysica Acta (BBA) - Bioenergetics, vol. 1797, no. 6-7, pp. 897-906, 2010.

[103] S. Dikalov, "Cross talk between mitochondria and NADPH oxidases," Free Radical Biology \& Medicine, vol. 51, no. 7, pp. 1289-1301, 2011.
[104] A. K. Doughan, D. G. Harrison, and S. I. Dikalov, "Molecular mechanisms of angiotensin II-mediated mitochondrial dysfunction: linking mitochondrial oxidative damage and vascular endothelial dysfunction," Circulation Research, vol. 102, no. 4, pp. 488-496, 2008.

[105] A. E. Dikalova, A. T. Bikineyeva, K. Budzyn et al., "Therapeutic targeting of mitochondrial superoxide in hypertension," Circulation Research, vol. 107, no. 1, pp. 106-116, 2010.

[106] D. B. Zorov, C. R. Filburn, L. O. Klotz, J. L. Zweier, and S. J. Sollott, "Reactive oxygen species (Ros-induced) Ros release: a new phenomenon accompanying induction of the mitochondrial permeability transition in cardiac myocytes," The Journal of Experimental Medicine, vol. 192, no. 7, pp. 10011014, 2000.

[107] A. E. Dikalova, H. A. Itani, R. R. Nazarewicz et al., "Sirt3 impairment and SOD2 hyperacetylation in vascular oxidative stress and hypertension," Circulation Research, vol. 121, no. 5, pp. 564-574, 2017.

[108] S. I. Dikalov and A. E. Dikalova, "Crosstalk between mitochondrial hyperacetylation and oxidative stress in vascular dysfunction and hypertension," Antioxidants \& Redox Signaling, 2019.

[109] S. Dikalov, H. Itani, B. Richmond et al., "Tobacco smoking induces cardiovascular mitochondrial oxidative stress, promotes endothelial dysfunction, and enhances hypertension," American Journal of Physiology-Heart and Circulatory Physiology, vol. 316, no. 3, pp. H639-H646, 2019.

[110] U. Forstermann and T. Munzel, "Endothelial nitric oxide synthase in vascular disease: from marvel to menace," Circulation, vol. 113, no. 13, pp. 1708-1714, 2006.

[111] T. Munzel, A. Daiber, V. Ullrich, and A. Mulsch, "Vascular consequences of endothelial nitric oxide synthase uncoupling for the activity and expression of the soluble guanylyl cyclase and the cGMP-dependent protein kinase," Arteriosclerosis, Thrombosis, and Vascular Biology, vol. 25, no. 8, pp. 15511557, 2005.

[112] V. Schachinger, M. B. Britten, and A. M. Zeiher, "Prognostic impact of coronary vasodilator dysfunction on adverse longterm outcome of coronary heart disease," Circulation, vol. 101, no. 16, pp. 1899-1906, 2000.

[113] S. Blankenberg, H. J. Rupprecht, C. Bickel et al., "Glutathione peroxidase 1 activity and cardiovascular events in patients with coronary artery disease," The New England Journal of Medicine, vol. 349, no. 17, pp. 1605-1613, 2003.

[114] B. Schottker, H. Brenner, E. H. Jansen et al., "Evidence for the free radical/oxidative stress theory of ageing from the CHANCES consortium: a meta-analysis of individual participant data," BMC Medicine, vol. 13, no. 1, p. 300, 2015.

[115] A. di Minno, L. Turnu, B. Porro et al., "8-Hydroxy-2-deoxyguanosine levels and cardiovascular disease: a systematic review and meta-analysis of the literature," Antioxidants \& Redox Signaling, vol. 24, no. 10, pp. 548-555, 2016.

[116] A. Fratta Pasini, A. Albiero, C. Stranieri et al., "Serum oxidative stress-induced repression of Nrf2 and GSH depletion: a mechanism potentially involved in endothelial dysfunction of young smokers," PLoS One, vol. 7, no. 1, article e30291, 2012.

[117] M. I. Yilmaz, M. Saglam, K. Caglar et al., "The determinants of endothelial dysfunction in CKD: oxidative stress and asymmetric dimethylarginine," American Journal of Kidney Diseases, vol. 47, no. 1, pp. 42-50, 2006. 
[118] B. Jurado-Gamez, M. C. Fernandez-Marin, J. L. Gomez-Chaparro et al., "Relationship of oxidative stress and endothelial dysfunction in sleep apnoea," European Respiratory Journal, vol. 37, no. 4, pp. 873-879, 2011.

[119] T. Munzel, T. Gori, R. M. Bruno, and S. Taddei, "Is oxidative stress a therapeutic target in cardiovascular disease?," European Heart Journal, vol. 31, no. 22, pp. 2741-2748, 2010.

[120] H. H. H. W. Schmidt, R. Stocker, C. Vollbracht et al., "Antioxidants in translational medicine," Antioxidants \& Redox Signaling, vol. 23, no. 14, pp. 1130-1143, 2015.

[121] K. T. Khaw, S. Bingham, A. Welch et al., "Relation between plasma ascorbic acid and mortality in men and women in EPIC-Norfolk prospective study: a prospective population study," The Lancet, vol. 357, no. 9257, pp. 657-663, 2001.

[122] A. Shuaib, K. R. Lees, P. Lyden et al., "NXY-059 for the treatment of acute ischemic stroke," The New England Journal of Medicine, vol. 357, no. 6, pp. 562-571, 2007.

[123] V. T.-V. Dao, A. I. Casas, G. J. Maghzal et al., "Pharmacology and clinical drug candidates in redox medicine," Antioxidants \& Redox Signaling, vol. 23, no. 14, pp. 1113-1129, 2015.

[124] A. I. Casas, V. T. V. Dao, A. Daiber et al., "Reactive oxygenrelated diseases: therapeutic targets and emerging clinical indications," Antioxidants \& Redox Signaling, vol. 23, no. 14, pp. 1171-1185, 2015.

[125] A. Cuadrado, G. Manda, A. Hassan et al., "Transcription factor NRF2 as a therapeutic target for chronic diseases: a systems medicine approach," Pharmacological Reviews, vol. 70, no. 2, pp. 348-383, 2018.

[126] D. Harman, "Aging: a theory based on free radical and radiation chemistry," Journal of Gerontology, vol. 11, no. 3, pp. 298-300, 1956.

[127] W. A. Waters, "Some recent developments in the chemistry of free radicals," Journal of the Chemical Society, pp. 409415, 1946.

[128] E. Cadenas and K. J. A. Davies, "Mitochondrial free radical generation, oxidative stress, and aging," Free Radical Biology \& Medicine, vol. 29, no. 3-4, pp. 222-230, 2000.

[129] G. Lenaz, C. Bovina, M. D'Aurelio et al., "Role of mitochondria in oxidative stress and aging," Annals of the New York Academy of Sciences, vol. 959, no. 1, pp. 199-213, 2002.

[130] G. C. Kujoth, A. Hiona, T. D. Pugh et al., "Mitochondrial DNA mutations, oxidative stress, and apoptosis in mammalian aging," Science, vol. 309, no. 5733, pp. 481-484, 2005.

[131] F. Yin, A. Boveris, and E. Cadenas, "Mitochondrial energy metabolism and redox signaling in brain aging and neurodegeneration," Antioxidants \& Redox Signaling, vol. 20, no. 2, pp. 353-371, 2014.

[132] B. van der Loo, M. Bachschmid, J. N. Skepper et al., “Ageassociated cellular relocation of Sod 1 as a self-defense is a futile mechanism to prevent vascular aging," Biochemical and Biophysical Research Communications, vol. 344, no. 3, pp. 972-980, 2006.

[133] V. I. Pérez, A. Bokov, H. V. Remmen et al., "Is the oxidative stress theory of aging dead?," Biochimica et Biophysica Acta (BBA) - General Subjects, vol. 1790, no. 10, pp. 1005-1014, 2009.

[134] R. M. Lebovitz, H. Zhang, H. Vogel et al., "Neurodegeneration, myocardial injury, and perinatal death in mitochondrial superoxide dismutase-deficient mice," Proceedings of the National Academy of Sciences of the United States of America, vol. 93, no. 18, pp. 9782-9787, 1996.
[135] Y. Li, T. T. Huang, E. J. Carlson et al., "Dilated cardiomyopathy and neonatal lethality in mutant mice lacking manganese superoxide dismutase," Nature Genetics, vol. 11, no. 4, pp. 376-381, 1995.

[136] A. B. Salmon, A. Richardson, and V. I. Perez, "Update on the oxidative stress theory of aging: does oxidative stress play a role in aging or healthy aging?" Free Radical Biology \& Medicine, vol. 48, no. 5, pp. 642-655, 2010.

[137] F. L. Muller, M. S. Lustgarten, Y. Jang, A. Richardson, and H. Van Remmen, "Trends in oxidative aging theories," Free Radical Biology \& Medicine, vol. 43, no. 4, pp. 477-503, 2007.

[138] D.-F. Dai, Y. Chiao, D. J. Marcinek, H. H. Szeto, and P. S. Rabinovitch, "Mitochondrial oxidative stress in aging and healthspan," Longevity \& Healthspan, vol. 3, no. 1, p. 6, 2014.

[139] R. T. Hamilton, M. E. Walsh, and H. Van Remmen, "Mouse models of oxidative stress indicate a role for modulating healthy aging," Journal of Clinical \& Experimental Pathology, vol. 1, Supplement 4, 2012.

[140] A. Berry and F. Cirulli, "The $p 66^{\text {Shc }}$ gene paves the way for healthspan: evolutionary and mechanistic perspectives," Neuroscience \& Biobehavioral Reviews, vol. 37, no. 5, pp. 790$802,2013$.

[141] J. Wanagat, D. F. Dai, and P. Rabinovitch, "Mitochondrial oxidative stress and mammalian healthspan," Mechanisms of Ageing and Development, vol. 131, no. 7-8, pp. 527-535, 2010.

[142] P. Wenzel, S. Schuhmacher, J. Kienhofer et al., "Manganese superoxide dismutase and aldehyde dehydrogenase deficiency increase mitochondrial oxidative stress and aggravate age-dependent vascular dysfunction," Cardiovascular Research, vol. 80, no. 2, pp. 280-289, 2008.

[143] M. Oelze, S. Kroller-Schon, S. Steven et al., "Glutathione peroxidase-1 deficiency potentiates dysregulatory modifications of endothelial nitric oxide synthase and vascular dysfunction in aging," Hypertension, vol. 63, no. 2, pp. 390396, 2014.

[144] S. M. Baumgartner-Parzer, L. Wagner, M. Pettermann, A. Gessl, and W. Waldhausl, "Modulation by high glucose of adhesion molecule expression in cultured endothelial cells," Diabetologia, vol. 38, no. 11, pp. 1367-1370, 1995.

[145] S. V. Brodsky, O. Gealekman, J. Chen et al., "Prevention and reversal of premature endothelial cell senescence and vasculopathy in obesity-induced diabetes by ebselen," Circulation Research, vol. 94, no. 3, pp. 377-384, 2004.

[146] J. Xu, Z. Xie, R. Reece, D. Pimental, and M. H. Zou, "Uncoupling of endothelial nitric oxidase synthase by hypochlorous acid: role of $\mathrm{NAD}(\mathrm{P}) \mathrm{H}$ oxidase-derived superoxide and peroxynitrite," Arteriosclerosis, Thrombosis, and Vascular Biology, vol. 26, no. 12, pp. 2688-2695, 2006.

[147] M. C. Desco, M. Asensi, R. Marquez et al., "Xanthine oxidase is involved in free radical production in type 1 diabetes: protection by allopurinol," Diabetes, vol. 51, no. 4, pp. 11181124, 2002.

[148] T. Heitzer, K. Krohn, S. Albers, and T. Meinertz, "Tetrahydrobiopterin improves endothelium-dependent vasodilation by increasing nitric oxide activity in patients with type II diabetes mellitus," Diabetologia, vol. 43, no. 11, pp. 1435-1438, 2000.

[149] N. J. Alp, S. Mussa, J. Khoo et al., “Tetrahydrobiopterindependent preservation of nitric oxide-mediated endothelial function in diabetes by targeted transgenic GTP- 
cyclohydrolase I overexpression," The Journal of Clinical Investigation, vol. 112, no. 5, pp. 725-735, 2003.

[150] T. Heitzer, B. Finckh, S. Albers, K. Krohn, A. Kohlschutter, and T. Meinertz, "Beneficial effects of $\alpha$-lipoic acid and ascorbic acid on endothelium-dependent, nitric oxide-mediated vasodilation in diabetic patients: relation to parameters of oxidative stress," Free Radical Biology \& Medicine, vol. 31, no. 1, pp. 53-61, 2001.

[151] G. Ye, N. S. Metreveli, R. V. Donthi et al., "Catalase protects cardiomyocyte function in models of type 1 and type 2 diabetes," Diabetes, vol. 53, no. 5, pp. 1336-1343, 2004.

[152] T. Nassar, B. Kadery, C. Lotan, N. Da'as, Y. Kleinman, and A. Haj-Yehia, "Effects of the superoxide dismutase-mimetic compound tempol on endothelial dysfunction in streptozotocin-induced diabetic rats," European Journal of Pharmacology, vol. 436, no. 1-2, pp. 111-118, 2002.

[153] D. G. Harrison, T. J. Guzik, H. E. Lob et al., "Inflammation, immunity, and hypertension," Hypertension, vol. 57, no. 2, pp. 132-140, 2011.

[154] P. M. Ridker, B. M. Everett, T. Thuren et al., "Antiinflammatory therapy with canakinumab for atherosclerotic disease," The New England Journal of Medicine, vol. 377, no. 12, pp. 1119-1131, 2017.

[155] P. Soltesz, G. Kerekes, H. Der et al., "Comparative assessment of vascular function in autoimmune rheumatic diseases: considerations of prevention and treatment," Autoimmunity Reviews, vol. 10, no. 7, pp. 416-425, 2011.

[156] G. Murdaca, B. M. Colombo, P. Cagnati, R. Gulli, F. Spano, and F. Puppo, "Endothelial dysfunction in rheumatic autoimmune diseases," Atherosclerosis, vol. 224, no. 2, pp. 309-317, 2012.

[157] G. A. Vena, M. Vestita, and N. Cassano, "Psoriasis and cardiovascular disease," Dermatologic Therapy, vol. 23, no. 2, pp. 144-151, 2010.

[158] A. E. Hak, E. W. Karlson, D. Feskanich, M. J. Stampfer, and K. H. Costenbader, "Systemic lupus erythematosus and the risk of cardiovascular disease: results from the nurses' health study," Arthritis \& Rheumatism, vol. 61, no. 10, pp. 13961402, 2009.

[159] M. J. L. Peters, D. P. M. Symmons, D. McCarey et al., "EULAR evidence-based recommendations for cardiovascular risk management in patients with rheumatoid arthritis and other forms of inflammatory arthritis," Annals of the Rheumatic Diseases, vol. 69, no. 2, pp. 325-331, 2010.

[160] J. Herrera, A. Ferrebuz, E. G. MacGregor, and B. RodriguezIturbe, "Mycophenolate mofetil treatment improves hypertension in patients with psoriasis and rheumatoid arthritis," Journal of the American Society of Nephrology, vol. 17, no. 12, Supplement 3, pp. S218-S225, 2006.

[161] A. Sodergren, K. Karp, K. Boman et al., "Atherosclerosis in early rheumatoid arthritis: very early endothelial activation and rapid progression of intima media thickness," Arthritis Research \& Therapy, vol. 12, no. 4, article R158, 2010.

[162] D. D. Balci, A. Balci, S. Karazincir et al., "Increased carotid artery intima-media thickness and impaired endothelial function in psoriasis," Journal of the European Academy of Dermatology and Venereology, vol. 23, no. 1, pp. 1-6, 2009.

[163] P. Libby, M. Nahrendorf, and F. K. Swirski, "Leukocytes link local and systemic inflammation in ischemic cardiovascular disease: an expanded "cardiovascular continuum"," Journal of the American College of Cardiology, vol. 67, no. 9, pp. 1091-1103, 2016.

[164] A. Di Cesare, P. Di Meglio, and F. O. Nestle, "The IL-23/Th17 axis in the immunopathogenesis of psoriasis," Journal of Investigative Dermatology, vol. 129, no. 6, pp. 1339-1350, 2009.

[165] C. Leonardi, R. Matheson, C. Zachariae et al., “Anti-interleukin-17 monoclonal antibody ixekizumab in chronic plaque psoriasis," The New England Journal of Medicine, vol. 366, no. 13, pp. 1190-1199, 2012.

[166] K. A. Papp, C. Leonardi, A. Menter et al., "Brodalumab, an anti-interleukin-17-receptor antibody for psoriasis," The New England Journal of Medicine, vol. 366, no. 13, pp. 1181-1189, 2012.

[167] J. C. Crispin and G. C. Tsokos, "IL-17 in systemic lupus erythematosus," Journal of Biomedicine and Biotechnology, vol. 2010, Article ID 943254, 4 pages, 2010.

[168] E. Choy, "Understanding the dynamics: pathways involved in the pathogenesis of rheumatoid arthritis," Rheumatology, vol. 51, Supplement 5, pp. v3-v11, 2012.

[169] V. Pasceri and E. T. H. Yeh, "A tale of two diseases: atherosclerosis and rheumatoid arthritis," Circulation, vol. 100, no. 21, pp. 2124-2126, 1999.

[170] B. W. Van Tassell, S. Toldo, E. Mezzaroma, and A. Abbate, "Targeting interleukin-1 in heart disease," Circulation, vol. 128, no. 17, pp. 1910-1923, 2013.

[171] J. M. M. Howson, W. Zhao, D. R. Barnes et al., "Fifteen new risk loci for coronary artery disease highlight arterial-wallspecific mechanisms," Nature Genetics, vol. 49, no. 7, pp. 1113-1119, 2017.

[172] D. Klarin, Q. M. Zhu, C. A. Emdin et al., "Genetic analysis in UK Biobank links insulin resistance and transendothelial migration pathways to coronary artery disease," Nature Genetics, vol. 49, no. 9, pp. 1392-1397, 2017.

[173] K. R. Paudel, N. Panth, and D. W. Kim, "Circulating endothelial microparticles: a key hallmark of atherosclerosis progression," Scientifica, vol. 2016, Article ID 8514056, 9 pages, 2016.

[174] J. Matsubara, S. Sugiyama, K. Sugamura et al., "A dipeptidyl peptidase-4 inhibitor, des-fluoro-sitagliptin, improves endothelial function and reduces atherosclerotic lesion formation in apolipoprotein E-deficient mice," Journal of the American College of Cardiology, vol. 59, no. 3, pp. 265-276, 2012.

[175] Z. Shah, T. Kampfrath, J. A. Deiuliis et al., "Long-term dipeptidyl-peptidase 4 inhibition reduces atherosclerosis and inflammation via effects on monocyte recruitment and chemotaxis," Circulation, vol. 124, no. 21, pp. 2338-2349, 2011.

[176] P. M. Ridker, B. M. Everett, A. Pradhan et al., "Low-dose methotrexate for the prevention of atherosclerotic events," The New England Journal of Medicine, vol. 380, no. 8, pp. 752-762, 2019.

[177] V. Guarner and M. E. Rubio-Ruiz, "Low-grade systemic inflammation connects aging, metabolic syndrome and cardiovascular disease," Interdisciplinary Topics in Gerontology, vol. 40, pp. 99-106, 2015.

[178] T. K. Howcroft, J. Campisi, G. B. Louis et al., "The role of inflammation in age-related disease," Aging, vol. 5, no. 1, pp. 84-93, 2013.

[179] A. C. Bulua, A. Simon, R. Maddipati et al., "Mitochondrial reactive oxygen species promote production of proinflammatory cytokines and are elevated in TNFR1-associated periodic 
syndrome (TRAPS)," Journal of Experimental Medicine, vol. 208, no. 3, pp. 519-533, 2011.

[180] A. P. West, I. E. Brodsky, C. Rahner et al., “TLR signalling augments macrophage bactericidal activity through mitochondrial ROS," Nature, vol. 472, no. 7344, pp. 476-480, 2011.

[181] R. Zhou, A. S. Yazdi, P. Menu, and J. Tschopp, “A role for mitochondria in NLRP3 inflammasome activation," Nature, vol. 469, no. 7329, pp. 221-225, 2011.

[182] R. Zhou, A. Tardivel, B. Thorens, I. Choi, and J. Tschopp, "Thioredoxin-interacting protein links oxidative stress to inflammasome activation," Nature Immunology, vol. 11, no. 2, pp. 136-140, 2010.

[183] R. R. Nazarewicz and S. I. Dikalov, "Mitochondrial ROS in the prohypertensive immune response," American Journal of Physiology-Regulatory, Integrative and Comparative Physiology, vol. 305, no. 2, pp. R98-R100, 2013.

[184] A. Martin-Montalvo, E. M. Mercken, S. J. Mitchell et al., "Metformin improves healthspan and lifespan in mice," Nature Communications, vol. 4, no. 1, 2013.

[185] D. G. Harrison, A. Vinh, H. Lob, and M. S. Madhur, "Role of the adaptive immune system in hypertension," Current Opinion in Pharmacology, vol. 10, no. 2, pp. 203-207, 2010.

[186] B. Rodriguez-Iturbe, H. Pons, and R. J. Johnson, "Role of the Immune system in hypertension," Physiological Reviews, vol. 97, no. 3, pp. 1127-1164, 2017.

[187] B. Rodriguez-Iturbe, H. Pons, Y. Quiroz, M. A. Lanaspa, and R. J. Johnson, "Autoimmunity in the pathogenesis of hypertension," Nature Reviews Nephrology, vol. 10, no. 1, pp. 5662, 2014.

[188] S. I. Dikalov, W. Li, A. K. Doughan, R. R. Blanco, and A. M. Zafari, "Mitochondrial reactive oxygen species and calcium uptake regulate activation of phagocytic NADPH oxidase," American Journal of Physiology-Regulatory, Integrative and Comparative Physiology, vol. 302, no. 10, pp. R1134-R1142, 2012.

[189] P. Wenzel, M. Knorr, S. Kossmann et al., "Lysozyme M-positive monocytes mediate angiotensin II-induced arterial hypertension and vascular dysfunction," Circulation, vol. 124, no. 12, pp. 1370-1381, 2011.

[190] S. Kossmann, M. Schwenk, M. Hausding et al., "Angiotensin II-induced vascular dysfunction depends on interferon- $\gamma$ driven immune cell recruitment and mutual activation of monocytes and NK-cells," Arteriosclerosis, Thrombosis, and Vascular Biology, vol. 33, no. 6, pp. 1313-1319, 2013.

[191] T. J. Guzik, N. E. Hoch, K. A. Brown et al., "Role of the T cell in the genesis of angiotensin II-induced hypertension and vascular dysfunction," Journal of Experimental Medicine, vol. 204, no. 10, pp. 2449-2460, 2007.

[192] C. T. Chan, C. G. Sobey, M. Lieu et al., "Obligatory role for B cells in the development of angiotensin II-dependent hypertension," Hypertension, vol. 66, no. 5, pp. 1023-1033, 2015.

[193] H. A. Itani, W. G. McMaster Jr., M. A. Saleh et al., “Activation of human T cells in hypertension: studies of humanized mice and hypertensive humans," Hypertension, vol. 68, no. 1, pp. 123-132, 2016.

[194] S. Kossmann, J. Lagrange, S. Jackel et al., "Platelet-localized FXI promotes a vascular coagulation-inflammatory circuit in arterial hypertension," Science Translational Medicine, vol. 9, no. 375, article eaah4923, 2017.
[195] A. Gasparyan, A. Stavropoulos-Kalinoglou, T. Toms, K. Douglas, and G. Kitas, "Association of mean platelet volume with hypertension in rheumatoid arthritis," Inflammation \& Allergy-Drug Targets, vol. 9, no. 1, pp. 45-50, 2010.

[196] A. Leader, D. Pereg, and M. Lishner, "Are platelet volume indices of clinical use? A multidisciplinary review," Annals of Medicine, vol. 44, no. 8, pp. 805-816, 2012.

[197] P. Wenzel, "Monocytes as immune targets in arterial hypertension," British Journal of Pharmacology, vol. 176, no. 12, pp. 1966-1977, 2019.

[198] M. Knorr, T. Munzel, and P. Wenzel, "Interplay of NK cells and monocytes in vascular inflammation and myocardial infarction," Frontiers in Physiology, vol. 5, p. 295, 2014.

[199] S. Karbach, J. Lagrange, and P. Wenzel, "Thromboinflammation and vascular dysfunction," Hämostaseologie, 2018.

[200] K. Pittman and P. Kubes, "Damage-associated molecular patterns control neutrophil recruitment," Journal of Innate Immunity, vol. 5, no. 4, pp. 315-323, 2013.

[201] E. Ortona, A. Maselli, F. Delunardo, T. Colasanti, A. Giovannetti, and M. Pierdominici, "Relationship between redox status and cell fate in immunity and autoimmunity," Antioxidants \& Redox Signaling, vol. 21, no. 1, pp. 103-122, 2014

[202] B. Brune, N. Dehne, N. Grossmann et al., "Redox control of inflammation in macrophages," Antioxidants \& Redox Signaling, vol. 19, no. 6, pp. 595-637, 2013.

[203] C. Janko, M. Filipovic, L. E. Munoz et al., "Redox modulation of HMGB1-related signaling," Antioxidants \& Redox Signaling, vol. 20, no. 7, pp. 1075-1085, 2014.

[204] A. Kirabo, V. Fontana, A. P. C. de Faria et al., "DC isoketalmodified proteins activate $\mathrm{T}$ cells and promote hypertension," The Journal of Clinical Investigation, vol. 124, no. 10, pp. 4642-4656, 2014.

[205] M. Qiao, Q. Zhao, C. F. Lee et al., “Thiol oxidative stress induced by metabolic disorders amplifies macrophage chemotactic responses and accelerates atherogenesis and kidney injury in LDL receptor-deficient mice," Arteriosclerosis, Thrombosis, and Vascular Biology, vol. 29, no. 11, pp. 17791786, 2009.

[206] H. S. Kim, S. L. Ullevig, D. Zamora, C. F. Lee, and R. Asmis, "Redox regulation of MAPK phosphatase 1 controls monocyte migration and macrophage recruitment," Proceedings of the National Academy of Sciences of the United States of America, vol. 109, no. 41, pp. E2803-E2812, 2012.

[207] H. S. Kim, S. L. Ullevig, H. N. Nguyen, D. Vanegas, and

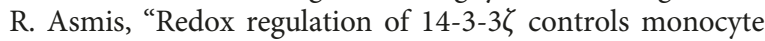
migration," Arteriosclerosis, Thrombosis, and Vascular Biology, vol. 34, no. 7, pp. 1514-1521, 2014.

[208] S. Ullevig, H. Kim, and R. Asmis, "S-glutathionylation in monocyte and macrophage (dys)function," International Journal of Molecular Sciences, vol. 14, no. 8, pp. 1521215232, 2013.

[209] C. Boeck, A. M. Koenig, K. Schury et al., "Inflammation in adult women with a history of child maltreatment: the involvement of mitochondrial alterations and oxidative stress," Mitochondrion, vol. 30, pp. 197-207, 2016.

[210] J. M. Abais, M. Xia, Y. Zhang, K. M. Boini, and P. L. Li, "Redox regulation of NLRP3 inflammasomes: ROS as trigger or effector?"” Antioxidants \& Redox Signaling, vol. 22, no. 13, pp. 1111-1129, 2015. 
[211] J. Y. Bae and H. H. Park, "Crystal structure of NALP3 protein pyrin domain (PYD) and its implications in inflammasome assembly," Journal of Biological Chemistry, vol. 286, no. 45, pp. 39528-39536, 2011.

[212] N. Maugeri, P. Rovere-Querini, M. Baldini et al., "Oxidative stress elicits platelet/leukocyte inflammatory interactions via HMGB1: a candidate for microvessel injury in sytemic sclerosis," Antioxidants \& Redox Signaling, vol. 20, no. 7, pp. 10601074, 2014.

[213] K. Stark, V. Philippi, S. Stockhausen et al., "Disulfide HMGB1 derived from platelets coordinates venous thrombosis in mice," Blood, vol. 128, no. 20, pp. 2435-2449, 2016.

[214] Y. Lei, K. Wang, L. Deng, Y. Chen, E. C. Nice, and C. Huang, "Redox regulation of inflammation: old elements, a new story," Medicinal Research Reviews, vol. 35, no. 2, pp. 306340, 2015.

[215] E. Venereau, M. Casalgrandi, M. Schiraldi et al., "Mutually exclusive redox forms of HMGB1 promote cell recruitment or proinflammatory cytokine release," Journal of Experimental Medicine, vol. 209, no. 9, pp. 1519-1528, 2012.

[216] E. P. Azevedo, N. C. Rochael, A. B. Guimaraes-Costa et al., "A metabolic shift toward pentose phosphate pathway is necessary for amyloid fibril- and phorbol 12-myristate 13acetate-induced neutrophil extracellular trap (NET) formation," Journal of Biological Chemistry, vol. 290, no. 36, pp. 22174-22183, 2015.

[217] J. Dan Dunn, L. A. Alvarez, X. Zhang, and T. Soldati, "Reactive oxygen species and mitochondria: a nexus of cellular homeostasis," Redox Biology, vol. 6, pp. 472-485, 2015.

[218] D. N. Douda, M. A. Khan, H. Grasemann, and N. Palaniyar, "SK3 channel and mitochondrial ROS mediate NADPH oxidase-independent NETosis induced by calcium influx," Proceedings of the National Academy of Sciences of the United States of America, vol. 112, no. 9, pp. 2817-2822, 2015.

[219] C. Lood, L. P. Blanco, M. M. Purmalek et al., "Neutrophil extracellular traps enriched in oxidized mitochondrial DNA are interferogenic and contribute to lupus-like disease," Nature Medicine, vol. 22, no. 2, pp. 146-153, 2016.

[220] M. Morshed, R. Hlushchuk, D. Simon et al., "NADPH oxidase-independent formation of extracellular DNA traps by basophils," The Journal of Immunology, vol. 192, no. 11, pp. 5314-5323, 2014.

[221] T. Kirchner, S. Moller, M. Klinger, W. Solbach, T. Laskay, and M. Behnen, "The impact of various reactive oxygen species on the formation of neutrophil extracellular traps," Mediators of Inflammation, vol. 2012, Article ID 849136, 10 pages, 2012.

[222] P. Wenzel, H. Rossmann, C. Muller et al., "Heme oxygenase1 suppresses a pro-inflammatory phenotype in monocytes and determines endothelial function and arterial hypertension in mice and humans," European Heart Journal, vol. 36, no. 48, pp. 3437-3446, 2015.

[223] C. K. Kramer, C. Ye, S. Campbell, and R. Retnakaran, "Comparison of new glucose-lowering drugs on risk of heart failure in type 2 diabetes: a network meta-analysis," JACC: Heart Failure, vol. 6, no. 10, pp. 823-830, 2018.

[224] G. P. Fadini, A. Avogaro, L. Degli Esposti et al., "Risk of hospitalization for heart failure in patients with type 2 diabetes newly treated with DPP-4 inhibitors or other oral glucoselowering medications: a retrospective registry study on 127,555 patients from the Nationwide OsMed Health-DB
Database," European Heart Journal, vol. 36, no. 36, pp. 2454-2462, 2015.

[225] K. B. Filion, L. Azoulay, R. W. Platt et al., "A multicenter observational study of incretin-based drugs and heart failure," The New England Journal of Medicine, vol. 374, no. 12, pp. 1145-1154, 2016.

[226] L. Timmers, J. P. S. Henriques, D. P. V. de Kleijn et al., "Exenatide reduces infarct size and improves cardiac function in a porcine model of ischemia and reperfusion injury," Journal of the American College of Cardiology, vol. 53, no. 6, pp. 501510, 2009.

[227] B. Hocher, Y. Sharkovska, M. Mark, T. Klein, and T. Pfab, "The novel DPP-4 inhibitors linagliptin and BI 14361 reduce infarct size after myocardial ischemia/reperfusion in rats," International Journal of Cardiology, vol. 167, no. 1, pp. 8793, 2013.

[228] I. Andreadou, P. Efentakis, E. Balafas et al., "Empagliflozin limits myocardial infarction in vivo and cell death in vitro: role of STAT3, mitochondria, and redox aspects," Frontiers in Physiology, vol. 8, p. 1077, 2017.

[229] H. Oshima, T. Miki, A. Kuno et al., "Empagliflozin, an SGLT2 inhibitor, reduced the mortality rate after acute myocardial infarction with modification of cardiac metabolomes and antioxidants in diabetic rats," Journal of Pharmacology and Experimental Therapeutics, vol. 368, no. 3, pp. 524-534, 2019.

[230] F. Bonnet and A. J. Scheen, "Effects of SGLT2 inhibitors on systemic and tissue low-grade inflammation: the potential contribution to diabetes complications and cardiovascular disease," Diabetes \& Metabolism, vol. 44, no. 6, pp. 457464, 2018.

[231] S. L. Atkin, N. Katsiki, M. Banach, D. P. Mikhailidis, M. Pirro, and A. Sahebkar, "Effect of dipeptidyl peptidase-4 inhibitors on circulating tumor necrosis factor- $\alpha$ concentrations: a systematic review and meta-analysis of controlled trials," Journal of Diabetes and its Complications, vol. 31, no. 9, pp. 1458-1464, 2017.

[232] Y. S. Lee and H. S. Jun, "Anti-inflammatory effects of GLP-1-based therapies beyond glucose control," Mediators of Inflammation, vol. 2016, Article ID 3094642, 11 pages, 2016.

[233] N. A. Alvarez-Villalobos, A. M. Trevino-Alvarez, and J. G. Gonzalez-Gonzalez, "Liraglutide and cardiovascular outcomes in type 2 diabetes," The New England Journal of Medicine, vol. 375, no. 18, pp. 1797-1798, 2016.

[234] S. P. Marso, S. C. Bain, A. Consoli et al., "Semaglutide and cardiovascular outcomes in patients with type 2 diabetes," The New England Journal of Medicine, vol. 375, no. 19, pp. 1834-1844, 2016.

[235] J. Lonborg, H. Kelbaek, N. Vejlstrup et al., "Exenatide reduces final infarct size in patients with ST-segment-elevation myocardial infarction and short-duration of ischemia," Circulation: Cardiovascular Interventions, vol. 5, no. 2, pp. 288295, 2012.

[236] V. A. Fonseca, J. H. Devries, R. R. Henry, M. Donsmark, H. F. Thomsen, and J. Plutzky, "Reductions in systolic blood pressure with liraglutide in patients with type 2 diabetes: insights from a patient-level pooled analysis of six randomized clinical trials," Journal of Diabetes and its Complications, vol. 28, no. 3, pp. 399-405, 2014.

[237] J. Koska, E. A. Schwartz, M. P. Mullin, D. C. Schwenke, and P. D. Reaven, "Improvement of postprandial 
endothelial function after a single dose of exenatide in individuals with impaired glucose tolerance and recentonset type 2 diabetes," Diabetes Care, vol. 33, no. 5, pp. 1028-1030, 2010.

[238] E. Akiyama, S. Sugiyama, J. Matsubara et al., "Decreased plasma levels of active glucagon-like peptide-1 in coronary artery disease," Journal of the American College of Cardiology, vol. 65, no. 7, pp. 754-755, 2015.

[239] D. S. Ghorpade, L. Ozcan, Z. Zheng et al., "Hepatocytesecreted DPP4 in obesity promotes adipose inflammation and insulin resistance," Nature, vol. 555, no. 7698, pp. 673677, 2018.

[240] X. Wang, M. Hausding, S. Y. Weng et al., "Gliptins suppress inflammatory macrophage activation to mitigate inflammation, fibrosis, oxidative stress, and vascular dysfunction in models of nonalcoholic steatohepatitis and liver fibrosis," Antioxidants \& Redox Signaling, vol. 28, no. 2, pp. 87-109, 2018.

[241] J. M. Petit and B. Verges, "GLP-1 receptor agonists in NAFLD," Diabetes \& Metabolism, vol. 43, pp. 2S28-2S33, 2017.

[242] B. M. Scirica, D. L. Bhatt, E. Braunwald et al., "Saxagliptin and cardiovascular outcomes in patients with type 2 diabetes mellitus," The New England Journal of Medicine, vol. 369, no. 14, pp. 1317-1326, 2013.

[243] M. M. Levy, A. Artigas, G. S. Phillips et al., "Outcomes of the Surviving Sepsis Campaign in intensive care units in the USA and Europe: a prospective cohort study," The Lancet Infectious Diseases, vol. 12, no. 12, pp. 919-924, 2012.

[244] H. C. Ku, W. P. Chen, and M. J. Su, "GLP-1 signaling preserves cardiac function in endotoxemic Fischer 344 and DPP4-deficient rats," Naunyn-Schmiedeberg's Archives of Pharmacology, vol. 382, no. 5-6, pp. 463-474, 2010.

[245] S. Kroller-Schon, M. Knorr, M. Hausding et al., "Glucoseindependent improvement of vascular dysfunction in experimental sepsis by dipeptidyl-peptidase 4 inhibition," Cardiovascular Research, vol. 96, no. 1, pp. 140-149, 2012.

[246] S. Steven, M. Hausding, S. Kroller-Schon et al., "Gliptin and GLP-1 analog treatment improves survival and vascular inflammation/dysfunction in animals with lipopolysaccharide-induced endotoxemia," Basic Research in Cardiology, vol. 110, no. 2, 2015.

[247] S. Steven, K. Jurk, M. Kopp et al., "Glucagon-like peptide-1 receptor signalling reduces microvascular thrombosis, nitrooxidative stress and platelet activation in endotoxaemic mice," British Journal of Pharmacology, vol. 174, no. 12, pp. 1620-1632, 2017.

[248] A. K. Gupta, A. K. Verma, J. Kailashiya, S. K. Singh, and N. Kumar, "Sitagliptin: anti-platelet effect in diabetes and healthy volunteers," Platelets, vol. 23, no. 8, pp. 565-570, 2012.

[249] A. Cameron-Vendrig, A. Reheman, M. A. Siraj et al., "Glucagon-like peptide 1 receptor activation attenuates platelet aggregation and thrombosis," Diabetes, vol. 65, no. 6, pp. 1714-1723, 2016.

[250] M. Levi and T. van der Poll, "Inflammation and coagulation," Critical Care Medicine, vol. 38, pp. S26-S34, 2010.

[251] A. Tahara, E. Kurosaki, M. Yokono et al., "Effects of SGLT2 selective inhibitor ipragliflozin on hyperglycemia, hyperlipidemia, hepatic steatosis, oxidative stress, inflammation, and obesity in type 2 diabetic mice," European Journal of Pharmacology, vol. 715, no. 1-3, pp. 246-255, 2013.
[252] A. Tahara, E. Kurosaki, M. Yokono et al., "Effects of sodiumglucose cotransporter 2 selective inhibitor ipragliflozin on hyperglycaemia, oxidative stress, inflammation and liver injury in streptozotocin-induced type 1 diabetic rats," Journal of Pharmacy and Pharmacology, vol. 66, no. 7, pp. 975-987, 2014.

[253] N. Terami, D. Ogawa, H. Tachibana et al., "Long-term treatment with the sodium glucose cotransporter 2 inhibitor, dapagliflozin, ameliorates glucose homeostasis and diabetic nephropathy in $d b / d b$ mice," PLoS One, vol. 9, no. 6, article e100777, 2014.

[254] Y. Nakatsu, H. Kokubo, B. Bumdelger et al., "The SGLT2 inhibitor luseogliflozin rapidly normalizes aortic mRNA levels of inflammation-related but not lipid-metabolismrelated genes and suppresses atherosclerosis in diabetic ApoE KO mice," International Journal of Molecular Sciences, vol. 18, no. 8, p. 1704, 2017.

[255] W. Leng, X. Ouyang, X. Lei et al., "The SGLT-2 inhibitor dapagliflozin has a therapeutic effect on atherosclerosis in diabetic $\mathrm{ApoE}^{-1-}$ mice," Mediators of Inflammation, vol. 2016, Article ID 6305735, 13 pages, 2016.

[256] J. H. Han, T. J. Oh, G. Lee et al., "The beneficial effects of empagliflozin, an SGLT2 inhibitor, on atherosclerosis in ApoE $E^{-1-}$ mice fed a western diet," Diabetologia, vol. 60, no. 2, pp. 364-376, 2017.

[257] S. L. Kabil and N. M. Mahmoud, "Canagliflozin protects against non-alcoholic steatohepatitis in type-2 diabetic rats through zinc alpha-2 glycoprotein up-regulation," European Journal of Pharmacology, vol. 828, pp. 135-145, 2018.

[258] C. Komiya, K. Tsuchiya, K. Shiba et al., "Ipragliflozin improves hepatic steatosis in obese mice and liver dysfunction in type 2 diabetic patients irrespective of body weight reduction," PLoS One, vol. 11, no. 3, article e0151511, 2016.

[259] Y. Honda, K. Imajo, T. Kato et al., "The selective SGLT2 inhibitor ipragliflozin has a therapeutic effect on nonalcoholic steatohepatitis in mice," PLoS One, vol. 11, no. 1, article e0146337, 2016.

[260] S. Qiang, Y. Nakatsu, Y. Seno et al., "Treatment with the SGLT2 inhibitor luseogliflozin improves nonalcoholic steatohepatitis in a rodent model with diabetes mellitus," Diabetology \& Metabolic Syndrome, vol. 7, no. 1, p. 104, 2015.

[261] Y. Hayashizaki-Someya, E. Kurosaki, T. Takasu et al., "Ipragliflozin, an SGLT2 inhibitor, exhibits a prophylactic effect on hepatic steatosis and fibrosis induced by cholinedeficient l-amino acid-defined diet in rats," European Journal of Pharmacology, vol. 754, pp. 19-24, 2015.

[262] T. Jojima, T. Tomotsune, T. Iijima, K. Akimoto, K. Suzuki, and Y. Aso, "Empagliflozin (an SGLT2 inhibitor), alone or in combination with linagliptin (a DPP-4 inhibitor), prevents steatohepatitis in a novel mouse model of non-alcoholic steatohepatitis and diabetes," Diabetology \& Metabolic Syndrome, vol. 8, no. 1, p. 45, 2016.

[263] T. Shibuya, N. Fushimi, M. Kawai et al., "Luseogliflozin improves liver fat deposition compared to metformin in type 2 diabetes patients with non-alcoholic fatty liver disease: a prospective randomized controlled pilot study," Diabetes, Obesity and Metabolism, vol. 20, no. 2, pp. 438-442, 2018.

[264] D. Ito, S. Shimizu, K. Inoue et al., "Comparison of ipragliflozin and pioglitazone effects on nonalcoholic fatty liver disease in patients with type 2 diabetes: a randomized, 24-week, open-label, active-controlled trial," Diabetes Care, vol. 40, no. 10, pp. 1364-1372, 2017. 
[265] L. Fala, "Jardiance (empagliflozin), an SGLT2 inhibitor, receives FDA approval for the treatment of patients with type 2 diabetes," American Health \& Drug Benefits, vol. 8, pp. 9295, 2015.

[266] T. Rieg, T. Masuda, M. Gerasimova et al., "Increase in SGLT1-mediated transport explains renal glucose reabsorption during genetic and pharmacological SGLT2 inhibition in euglycemia," American Journal of Physiology-Renal Physiology, vol. 306, no. 2, pp. F188-F193, 2014.

[267] M. Oelze, S. Kroller-Schon, P. Welschof et al., "The sodiumglucose co-transporter 2 inhibitor empagliflozin improves diabetes-induced vascular dysfunction in the streptozotocin diabetes rat model by interfering with oxidative stress and glucotoxicity," PLoS One, vol. 9, no. 11, article e112394, 2014.

[268] A. J. Scheen and N. Paquot, "Metabolic effects of SGLT-2 inhibitors beyond increased glucosuria: a review of the clinical evidence," Diabetes \& Metabolism, vol. 40, no. 6, Supplement 1, pp. S4-S11, 2014.

[269] J. L. Evans and I. D. Goldfine, "Aging and insulin resistance: just say iNOS,” Diabetes, vol. 62, no. 2, pp. 346-348, 2013.

[270] B. Zinman, C. Wanner, J. M. Lachin et al., "Empagliflozin, cardiovascular outcomes, and mortality in type 2 diabetes," The New England Journal of Medicine, vol. 373, no. 22, pp. 2117-2128, 2015.

[271] D. Fitchett, B. Zinman, C. Wanner et al., "Heart failure outcomes with empagliflozin in patients with type 2 diabetes at high cardiovascular risk: results of the EMPA-REG OUTCOME $^{\circledR}$ trial," European Heart Journal, vol. 37, no. 19, pp. 1526-1534, 2016.

[272] S. Steven, M. Oelze, A. Hanf et al., "The SGLT2 inhibitor empagliflozin improves the primary diabetic complications in ZDF rats," Redox Biology, vol. 13, pp. 370-385, 2017.

[273] A. Jufvas, S. Sjodin, K. Lundqvist, R. Amin, A. V. Vener, and P. Stralfors, "Global differences in specific histone H3 methylation are associated with overweight and type 2 diabetes," Clinical Epigenetics, vol. 5, no. 1, p. 15, 2013.

[274] F. Miao, D. D. Smith, L. Zhang, A. Min, W. Feng, and R. Natarajan, "Lymphocytes from patients with type 1 diabetes display a distinct profile of chromatin histone H3 lysine 9 dimethylation: an epigenetic study in diabetes," Diabetes, vol. 57, no. 12, pp. 3189-3198, 2008.

[275] E. Lubos, N. J. Kelly, S. R. Oldebeken et al., "Glutathione peroxidase-1 deficiency augments proinflammatory cytokine-induced redox signaling and human endothelial cell activation," Journal of Biological Chemistry, vol. 286, no. 41, pp. 35407-35417, 2011.

[276] R. S. Esworthy, R. Aranda, M. G. Martin, J. H. Doroshow, S. W. Binder, and F. F. Chu, "Mice with combined disruption of Gpx1 and Gpx2 genes have colitis," American Journal of Physiology-Gastrointestinal and Liver Physiology, vol. 281, no. 3, pp. G848-G855, 2001.

[277] K. Murakami, N. Murata, Y. Noda et al., "SOD1 (copper/zinc superoxide dismutase) deficiency drives amyloid $\beta$ protein oligomerization and memory loss in mouse model of Alzheimer disease," Journal of Biological Chemistry, vol. 286, no. 52, pp. 44557-44568, 2011.

[278] M. J. Kwon, Y. J. Jeon, K. Y. Lee, and T. Y. Kim, “Superoxide dismutase 3 controls adaptive immune responses and contributes to the inhibition of ovalbumin-induced allergic airway inflammation in mice," Antioxidants \& Redox Signaling, vol. 17, no. 10, pp. 1376-1392, 2012.
[279] T. Jansen, S. Kroller-Schon, T. Schonfelder et al., " $\alpha 1$ AMPK deletion in myelomonocytic cells induces a proinflammatory phenotype and enhances angiotensin IIinduced vascular dysfunction," Cardiovascular Research, vol. 114, no. 14, pp. 1883-1893, 2018.

[280] S. Kröller-Schön, T. Jansen, T. L. P. Tran et al., "Endothelial $\alpha 1 \mathrm{AMPK}$ modulates angiotensin II-mediated vascular inflammation and dysfunction," Basic Research in Cardiology, vol. 114, no. 2, 2019.

[281] C. E. Murdoch, S. Chaubey, L. Zeng et al., "Endothelial NADPH oxidase-2 promotes interstitial cardiac fibrosis and diastolic dysfunction through proinflammatory effects and endothelial-mesenchymal transition," Journal of the American College of Cardiology, vol. 63, no. 24, pp. 2734-2741, 2014.

[282] S. Kroller-Schon, A. Daiber, S. Steven et al., "Crucial role for Nox2 and sleep deprivation in aircraft noise-induced vascular and cerebral oxidative stress, inflammation, and gene regulation," European Heart Journal, vol. 39, no. 38, pp. 3528-3539, 2018.

[283] S. Liang, H. Y. Ma, Z. Zhong et al., "NADPH oxidase 1 in liver macrophages promotes inflammation and tumor development in mice," Gastroenterology, vol. 156, no. 4, pp. 11561172.e6, 2019.

[284] L. Thomson, M. Tenopoulou, R. Lightfoot et al., "Immunoglobulins against tyrosine-nitrated epitopes in coronary artery disease," Circulation, vol. 126, no. 20, pp. 2392-2401, 2012. 


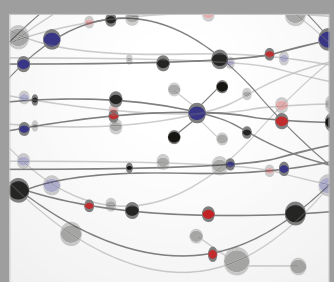

The Scientific World Journal
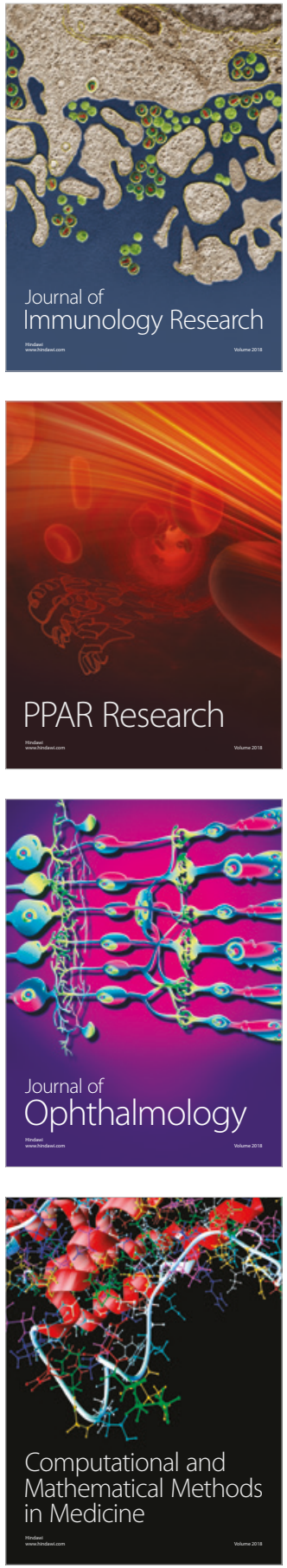

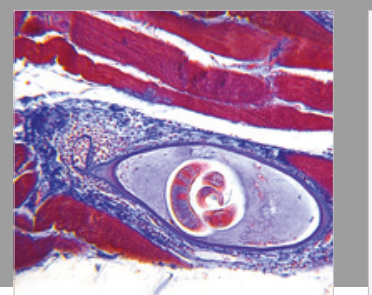

Gastroenterology Research and Practice

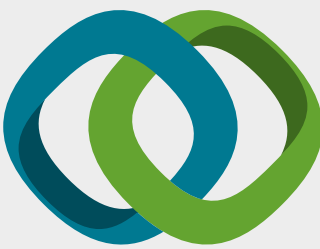

\section{Hindawi}

Submit your manuscripts at

www.hindawi.com
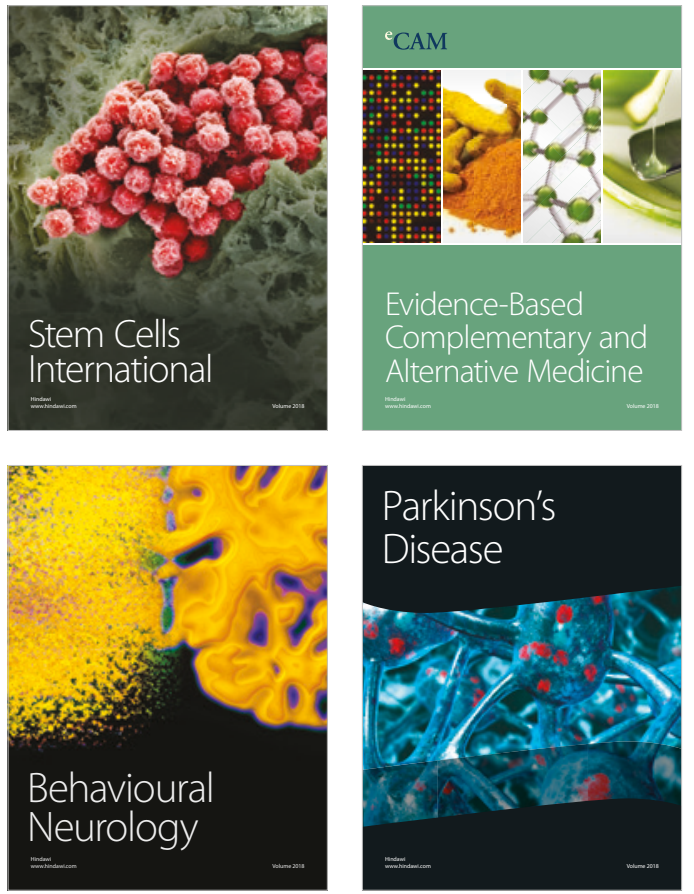

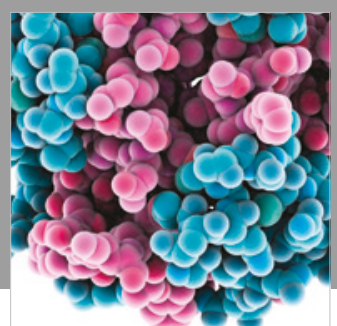

ournal of

Diabetes Research

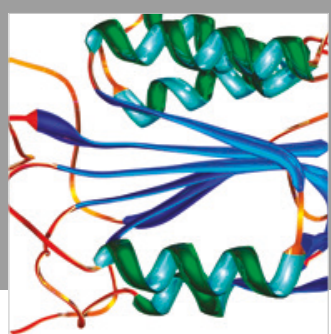

Disease Markers
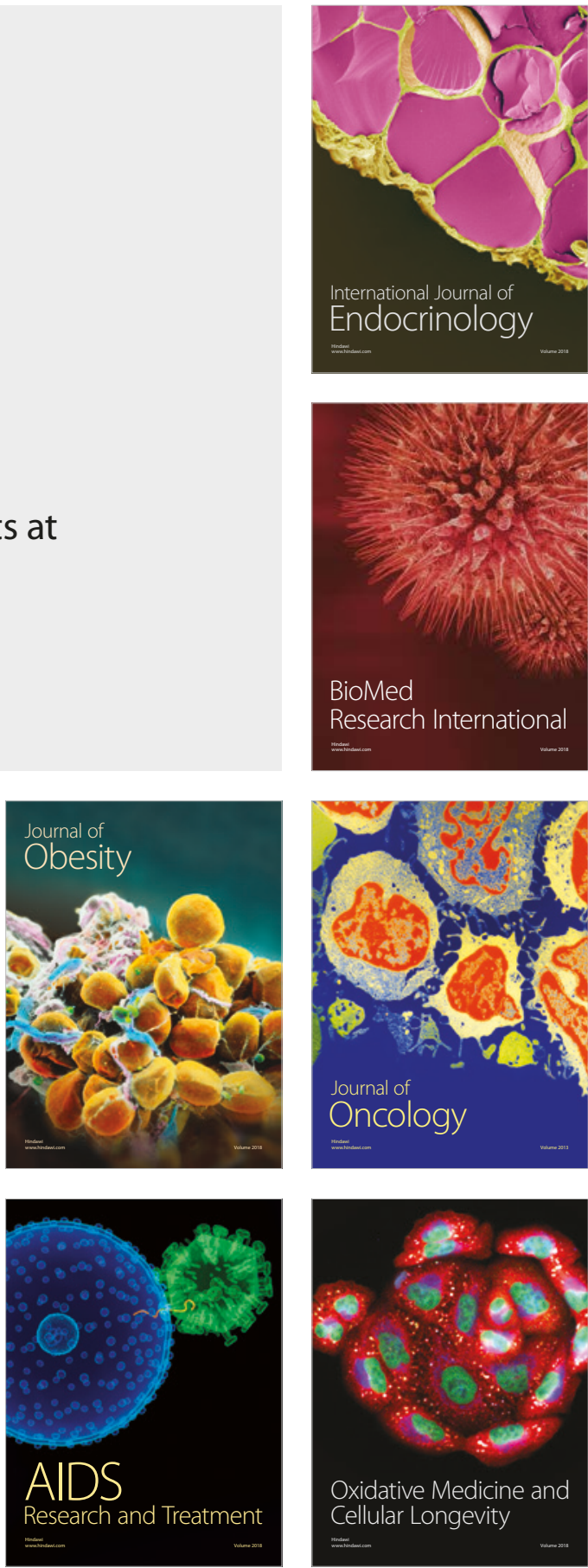